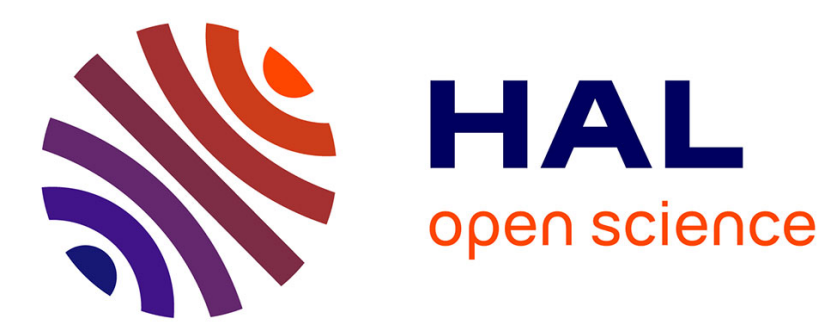

\title{
Fluid-structure interaction of a flexible rotor in water
}

Ahmed S Eldemerdash, T. Leweke

\section{To cite this version:}

Ahmed S Eldemerdash, T. Leweke. Fluid-structure interaction of a flexible rotor in water. Journal of Fluids and Structures, 2021, 103, pp.103259. 10.1016/j.jfluidstructs.2021.103259 . hal-03344067

\section{HAL Id: hal-03344067 \\ https://hal.science/hal-03344067}

Submitted on 5 Nov 2021

HAL is a multi-disciplinary open access archive for the deposit and dissemination of scientific research documents, whether they are published or not. The documents may come from teaching and research institutions in France or abroad, or from public or private research centers.
L'archive ouverte pluridisciplinaire HAL, est destinée au dépôt et à la diffusion de documents scientifiques de niveau recherche, publiés ou non, émanant des établissements d'enseignement et de recherche français ou étrangers, des laboratoires publics ou privés. 


\title{
Fluid-structure interaction of a flexible rotor in water
}

\author{
A.S. Eldemerdash, T. Leweke* \\ Aix-Marseille Université, CNRS, Centrale Marseille, IRPHE, 13384 Marseille, France
}

\begin{abstract}
In this study, a moderately flexible rotor is investigated experimentally in a water channel. The rotor consists of a single blade with a simplified rectangular geometry and is tested with various pitch angles at different rotational speeds. The flapwise bending deformation and induced torsion of the blade are measured using image processing tools, and flow fields are extracted with Particle Image Velocimetry. The blade deformation behaviour depends strongly on the pitch angle, it includes extreme downstream and upstream bending for high negative and positive pitch, respectively. In addition, large-amplitude, low-frequency bending fluctuations are observed in a certain range of rotational speeds for negative pitch. The wake vorticity fields show large-scale recirculation zones being formed and intermittently shed behind the rotor, resembling the dynamics of the Vortex Ring State known from helicopter aerodynamics. A comparison with the flow generated by a rigid rotor of the same geometry is also carried out.
\end{abstract}

Keywords: Flexible rotor, Fluid-Structure Interaction, Particle Image Velocimetry, Digital Image Correlation, Vortex Ring State

\section{Introduction}

Rotors are used in a wide variety of applications, including wind turbines (Hansen, 2015), helicopters (Leishman, 2006) and propellers (Wald, 2006). The blades of these rotors are often flexible, e.g. those of a large wind turbine bending under wind loads or their own weight, or those of a drone made of flexible material for reasons of safety and cost. Flexibility can be detrimental to rotor operation, since it can lead to excessive bending of the blade, which can then break or collide with the tower structure of a wind turbine, or to various unwanted instabilities of the fluidstructure interaction: static divergence, classical flutter or luffing (Winston, 1968a,b). Flexibility can, however, also be beneficial. It has been investigated as a means for increasing the mechanical strength of a rotor, or for expanding its range of operation by passively adapting to changes in the external environment. Examples are found in the work of MacPhee and Beyene (2015) and Cognet et al. (2017), who studied the effect of passive pitch modifications and chordwise bending, respectively, for enhancing the efficiency and operational envelope of a wind turbine rotor. Sicard and Sirohi (2012) performed experiments with an extremely flexible rotor intended for use on a micro air vehicle (MAV). Its blades could potentially be rolled up and stowed in the hub, allowing for an adjustable rotor diameter during operation, in addition to a lower weight and higher resistance against permanent damage after a collision. Lv et al. (2015) designed and tested a flexible rotor for a convertible MAV, where the twist distribution passively adapted to each flow regime for an overall performance enhancement. MAVs, and in particular rotor driven devices, have become increasingly popular over the past two decades, due to their versatility in civil and military applications, including surveillance and exploration of confined spaces. The development of more efficient rotors for these vehicles, taking into account the flexibility of the blades and potentially using it in a beneficial way, is an active field of research. A recent modelling study aimed at the optimisation of flexible rotors, including also the structure of its wake, was carried out by Durán Venegas et al. (2019).

According to Winston (1968b) and Sicard and Sirohi (2012), flexible rotors can be grouped into different categories, depending on the flexibility of the material. The first type is a moderately flexible rotor, in which the blades

\footnotetext{
${ }^{*}$ Corresponding author.

Email address: Thomas. Leweke@univ-amu.fr (T. Leweke)
} 
can sustain their own weight without rotation. The second type is a highly flexible rotor, whose blades cannot sustain their weight at rest, and a tip mass is generally needed to stretch the blade during rotation through centrifugal forces. Such a mass can also be used to control the blade twist. The third type is a fully flexible rotor, in which the blades have an additional flexibility in the chordwise direction, potentially allowing for a luffing instability to occur. In the present study, we consider small-scale rotors of moderate flexibility, admitting mostly flapwise bending deformations of the blades and, to a lesser degree, blade twist.

The rotors investigated in this work operate in water, where fluid forces are considerably higher than in air and lead to much larger deformations. In addition to the fundamental interest of these strong fluid-structure interactions, this configuration may have relevance for the recent development of so-called AquaUAVs (aquatic unmanned aerial vehicles, see Siddall and Kovač, 2014; Yang et al., 2015). In particular, Tan et al. (2017) have designed and tested an AquaUAV using a single propeller for operation in both air and water.

In the following, we present the results of an experimental study of a moderately flexible rotor in water. The rotor has a simple generic geometry, consisting of a single untwisted rectangular blade of constant thickness. The experimental set-up and the methods used for measuring the blade deformations and flow field are described in section 2 . Section 3 presents a survey of the observed bending and twist deformations, as function of the blade pitch and of the rotation frequency of the rotor, followed in section 4 by measurements of the velocity and vorticity fields characterising the corresponding rotor wake structures. In section 5, an intriguing phenomenon involving large-amplitude, low-frequency bending fluctuations is analysed in more detail and linked to a particular regime of rotor operation known from helicopter aerodynamics. Finally, comparisons with measurements made for rigid rotors having the same geometry are shown in section 6 , in order to assess the effect of flexibility on the flow structure and rotor performance (thrust). Conclusions are given in section 7.

\section{Experimental details}

\subsection{Set-up}

Experiments were performed in a hydrodynamic channel with a recirculating free-surface water flow of low turbulence intensity $(<1 \%)$. The test section has dimensions of $38 \mathrm{~cm}$ width, $50 \mathrm{~cm}$ height and $150 \mathrm{~cm}$ length, and the water height in the channel is $45 \mathrm{~cm}$. A stepper motor is used to drive a rotor mounted on a carbon-fibre shaft of $1.5 \mathrm{~cm}$ diameter with an ogive-shaped tip, through a gear box, as shown in Fig. 1(a). The rotor has a radius $R_{0}=88 \mathrm{~mm}$ and consists of a single untwisted rectangular blade of constant chord $c=20 \mathrm{~mm}$. The blade has a rectangular flat plate profile with a constant thickness of $0.7 \mathrm{~mm}$ (3.5\% of the chord), and with blunt leading and trailing edges. The various rotor dimensions are summarized in Fig. 2(a). Different blade pitch angles are tested. For each pitch, a separate hub is manufactured from nylon by rapid prototyping (Fig. 1b). The blade is made of low-density polyethylene (LDPE), whose material properties, shown in Table 1, were determined from controlled bending and twisting experiments (with an error of $\sim 10 \%$ ). The blade is attached to the hub and is replaced after each set of experiments, due to the permanent damage it undergoes during operation. For the purpose of comparison, a rigid blade of the same planform dimensions and thickness $1 \mathrm{~mm}$ (5\% of the chord), made of stainless steel, was also used in this study.

A cylindrical coordinate system is used in the following, with the origin at the centre of the undeformed rotor; the axial (downstream) distance is $z$, and the radial coordinate is $r$ (in some cases where an entire rotor wake cross section is shown, a coordinate $y$ is used). The various angles describing the blade geometry and the relative flow with respect to sections at different radial positions are illustrated in Fig. 2(b). Since the blade has a rectangular flat-plate profile without distinction between leading and trailing edges, and the rotation axis is aligned with the centre-chord line, a change of sign of the pitch angle can be obtained by simply inverting the direction of rotation. The velocity $U$ in the axial direction is the sum of the incoming free-stream velocity $U_{0}$ and the rotor-induced axial velocity $u_{i}$. The azimuthal velocity $V$ includes the component $2 \pi r f$ due to the blade rotation with frequency $f$, and the induced

Table 1

\begin{tabular}{lll}
\multicolumn{2}{l}{ Blade material - low-density polyethylene (LDPE). } \\
\hline Property & Symbol & Value \\
\hline Young's modulus & $E$ & $(3.5 \pm 0.3) \mathrm{GPa}$ \\
Shear modulus & $G$ & $(2.1 \pm 0.2) \mathrm{GPa}$ \\
Density & $\rho$ & $1070 \mathrm{~kg} / \mathrm{m}^{3}$ \\
\hline
\end{tabular}



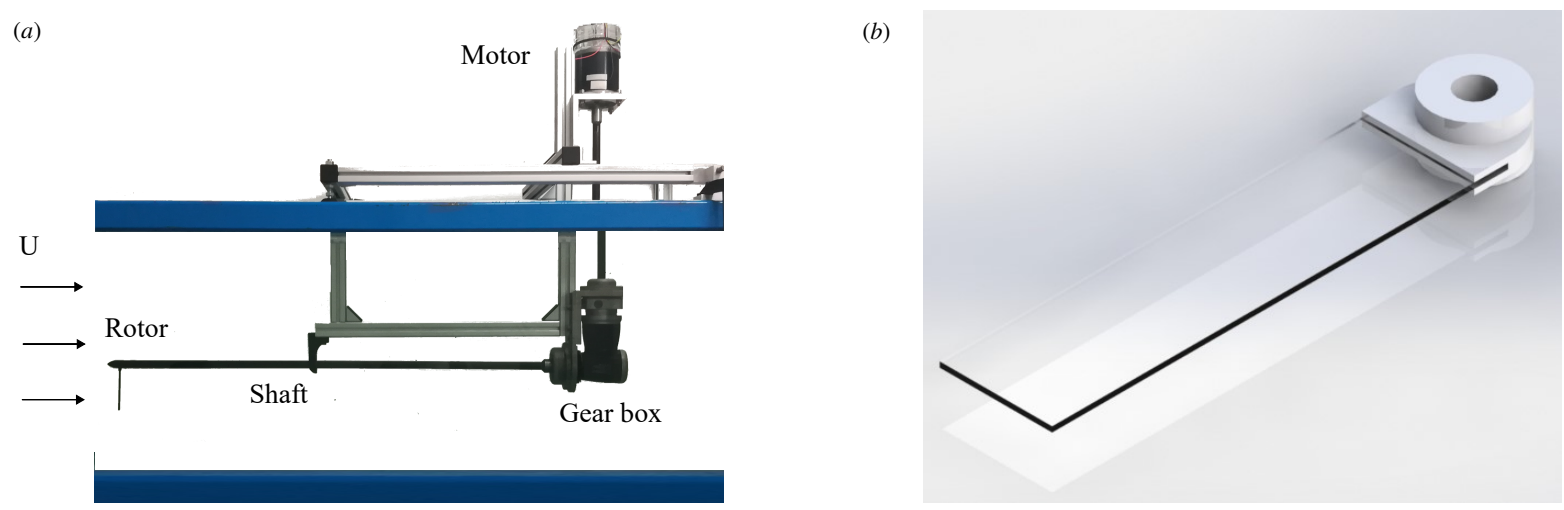

Fig. 1. (a) Experimental set-up (water channel test section, side view). (b) Three-dimensional schematic of the blade and hub.

(a)

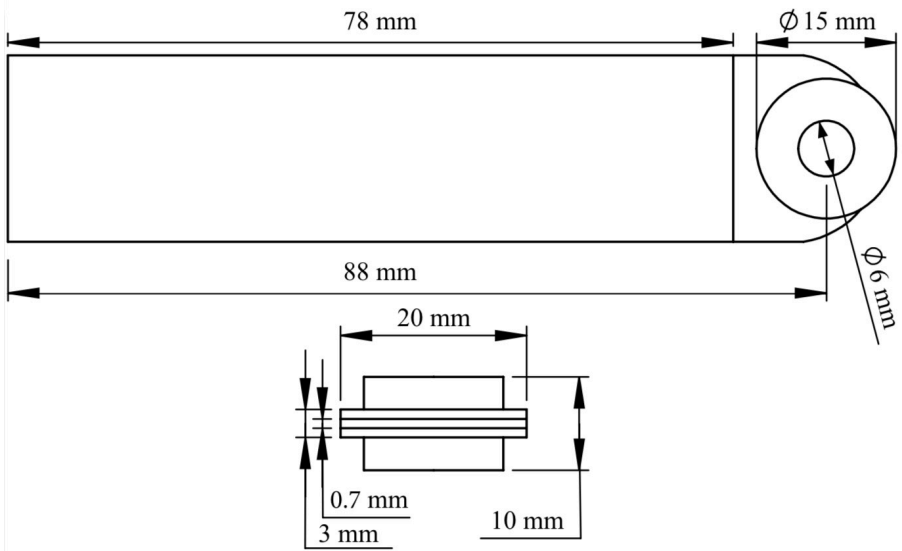

(b)

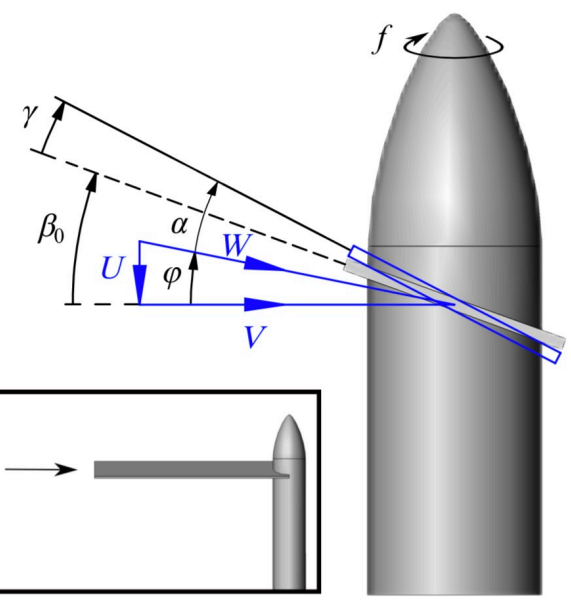

Fig. 2. (a) Blade and hub dimensions for a blade with $\beta_{0}=0^{\circ}$. (b) Blade angles and relative velocities. $\beta_{0}$ : pitch, $\gamma$ : twist, $\varphi$ : flow angle, $\alpha$ : angle of attack. The inset shows the view direction. The blue contour represents the twisted blade position.

azimuthal velocity $\mathrm{v}_{i}$. The total velocity seen by the blade has a magnitude $W=\sqrt{U^{2}+V^{2}}$ and is oriented at an angle $\varphi=\tan ^{-1}(U / V)$ with respect to the rotor plane. If $\gamma$ is the local induced twist angle (deviation from the pitch angle $\beta_{0}$ ) of the flexible blade, the effective (aerodynamic) angle of attack $\alpha$ at a given blade section is

$$
\alpha=\beta_{0}+\gamma-\varphi
$$

\subsection{Measurement techniques}

\subsubsection{Flow field}

Two-component Particle Image Velocimetry (PIV) was used to extract the velocity and vorticity fields in a plane containing the rotor axis. The fluid in the channel was seeded by $10 \mu \mathrm{m}$ silver-coated glass particles and illuminated using a Nd:YAG pulsed laser (Quantel Twins Ultra 190) positioned below the channel (Fig. 3a). Taking into account the symmetry of the flow, most measurement only include one half of the rotor centre plane. For certain cases, the other half was also measured; for this, a mirror was placed on top of a transparent acrylic plate positioned at the free surface, in order to illuminate this region.

Particle images were recorded using a high-resolution digital camera (Roper Redlake). It was triggered using the stepper motor's feedback for phase-averaging, or by an internal trigger for time-averaging. Phase-averaging was performed by averaging a number of flow fields with the blade at the same position, typically within the measurement plane, for a given set of rotor and flow parameters. Time-averaging was obtained by averaging flow fields recorded 

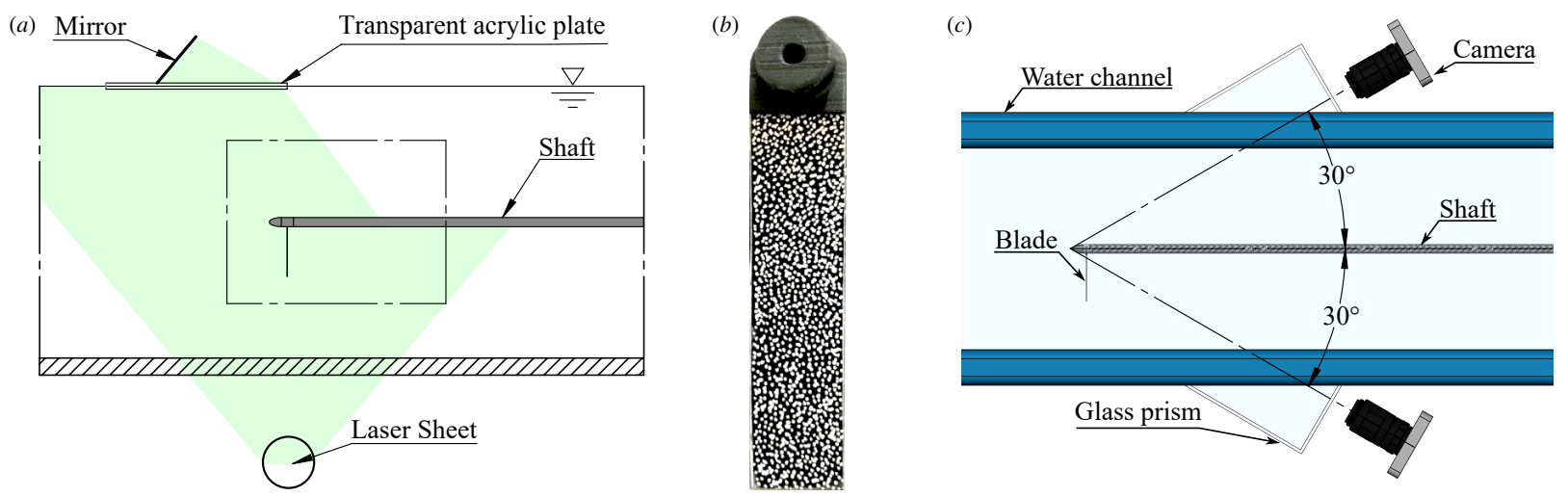

Fig. 3. (a) Schematic for the PIV measurements. (b) Blade with speckle pattern. (c) Schematic top view of the DIC set-up.

for different blade positions, scanning one or more complete rotor revolutions. The number of fields used for phase and time averaging was 200 for most of the cases, except the ones with large-amplitude ending fluctuating, for which 500 fields where used. Mean values of the velocities typically converged to within $1 \%$ of the final value using 100 and 200 fields, respectively, for these cases. Longer sequences of 1200 fields were also recorded for a more detailed characterisation of the unsteady bending behaviour. PIV image analysis and post-processing were performed with an in-house MATLAB code (see Meunier and Leweke, 2003).

\subsubsection{Deformation}

The deformations of the rotating flexible blade can generally be classified as follows: flapwise bending, in the direction parallel to the rotor axis; lead-lag bending, an azimuthal deformation in the rotor plane; chordwise deformation, which affects the blade profile geometry (e.g., a change of profile camber sign, for the case of a luffing instability); and torsion, i.e. the induced blade twist. In this study, only flapwise bending and torsion are measured. Lead-lag bending and chordwise deformations can be neglected, due to the high blade stiffness in these directions.

Commonly used methods for determining the deformation of a moving object can be divided into two categories. The first employs sensors (e.g., strain gauges) that detect surface deformations (e.g., Tang and Dowell, 1993). The second relies on optical techniques (e.g., Graves and Burner, 2001; Boden and Stasicki, 2014) and were found to be more appropriate for this study, due to the higher resolution and lower complexity in the set-up, compared to the first method, especially for small-scale rotors.

Two optical techniques were used to extract the blade deformation. Flapwise bending was obtained by processing side-view images of the blade captured during rotation. For a given set of flow, blade and rotation parameters, a number of images (between 50 and 400) were recorded at the same phase. From the superpositions of these snapshots (Figs. 5 and 6), the mean amplitude and range of flapwise bending could be determined, as shown in section 3.1.

The second technique involves three-dimensional Digital Image Correlation (DIC), which is here used to determine the induced twist distribution of the rotating blade. DIC refers to a class of non-contacting methods, which perform image analysis of an object, in order to capture its shape, deformation and motion (Sutton et al., 2009). Using random speckle patterns on the object's surface, images captured from different directions and/or at different times are matched, and comparing the speckles in small regions of the images allows the extraction of the full-field deformation in a given region of interest. Three-dimensional (3D) DIC has recently been used by Sicard and Sirohi (2013) and Uehara and Sirohi (2019) to accurately measure the deformation of rotating flexible blades. In the present study, a method based on the open-source MATLAB package MultiDIC, developed by Solav et al. (2018) at MIT, was employed to measure deformations, and in particular the blade twist.

3D DIC is here performed on stereo images captured by two cameras positioned on opposite sides of the rotor axis, as shown in Fig. 3(c). The rotor blade surface is spray-painted in black, and random white speckles are added manually with a correction fluid pen (Fig. 3b). The blade is illuminated from the downstream direction with the light from a pulsed Nd-YAG laser. The cameras and laser are triggered using the stepper motor feedback. In order to correct refraction effects, water-filled glas prisms are fixed to the side walls of the channel. Prior to the blade deformation 

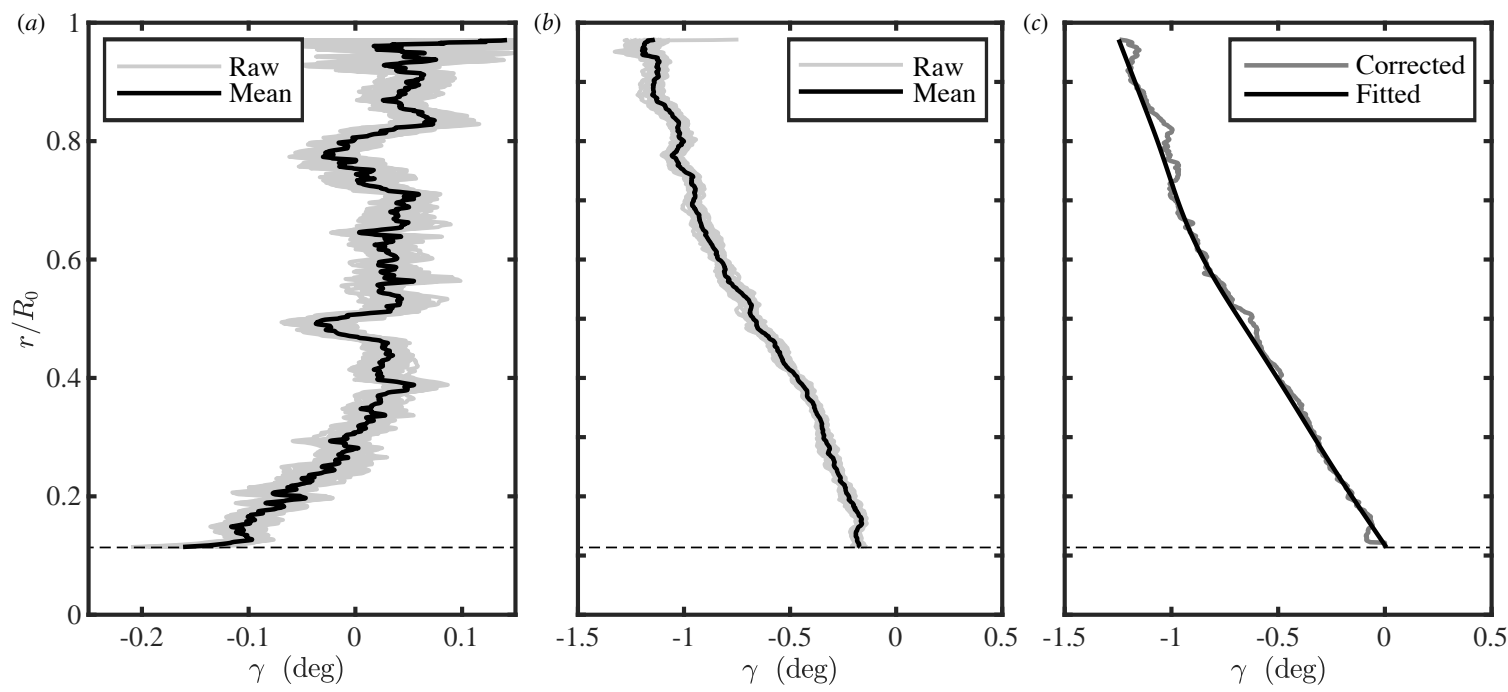

Fig. 4. Processing of DIC data for the determination of the blade twist distribution. (a) Measurement for the stationary blade without flow. (b) Measurement for the rotating blade with $\beta_{0}=5^{\circ}, f=2 \mathrm{~Hz}$ and $U_{0}=18 \mathrm{~cm} / \mathrm{s}$. (c) Data of (b) corrected with (a) and smoothly fitted, imposing $\gamma(r=0)=0$.

measurements, the distortion parameters for each camera are calculated using a checkerboard image, and a calibration is performed with a three-dimensional calibration target in the form of a cylinder, whose surface is covered with a dot pattern of known 3D coordinates. During the rotor experiments, phase-locked stereo images are then recorded, and the three-dimensional shape of the blade is determined by the MultiDIC algorithm, using the distortion and calibration parameters. The correlation algorithm provides a matrix of points in three dimensions, representing the downstream surface of the blade containing the speckle pattern. Identifying the leading and trailing edges allows the calculation of the orientation in space of chord sections along the blade span, from which the twist angles can be deduced using the geometrical relations shown in Fig. 2(b).

Figure 4 summarises the procedure used to process the raw twist data obtained in this way. For each test case, 100 image pairs were recorded at the same phase of the blade rotation, and the corresponding individual (raw) twist distributions were averaged. For a given blade, a reference case without rotation and without flow in the channel, for which the twist is known to be zero everywhere, was treated first, in order to evaluate the measurement scatter and error. Figure 4(a) shows the result for the blade with pitch $\beta_{0}=5^{\circ}$. The scatter of the individual measurements (gray lines) is less than $\pm 0.05^{\circ}$, and the error of the mean twist (black line) is below $\pm 0.1^{\circ}$, showing the high accuracy of the DIC method. The remaining error of the mean twist is possibly linked to the random variations of the speckle pattern along the blade, as well as to changes in the illumination and contrast. The overall uncertainty compares well with the one obtained by Sicard and Sirohi (2013) in a similar setting. For the twist measurement of a rotating blade in a free stream, the raw data from the phase-locked image pairs was again averaged (Fig. 4b), and the mean value from the measurements without rotation was subtracted from this average, in order to correct part of the systematic error. Finally, the resulting distribution was fitted to a smooth curve, imposing a vanishing twist at the junction with the hub (Fig. 4c).

With the set-up shown in Fig. 3(c) using two cameras, simultaneous images of the entire blade could be captured only for moderate flapwise bending deformations $\left(|\theta| \lesssim 30^{\circ}\right)$, since for higher blade deformation angle, part of the blade surface becomes concealed from at least one of the two cameras. Higher deformations can in principle also be treated, using a different set-up with more than two cameras; this was not pursued in the present study.

In the following sections, the deformation and flow field measurements of the rotor blades are presented. For this study, a constant free-stream velocity $U_{0}=18 \mathrm{~cm} / \mathrm{s}$ was chosen, and the rotation frequency of the rotor was varied in the range $0<f<18 \mathrm{~Hz}$. This corresponds to tip speed ratios $\lambda=2 \pi f R_{0} / U_{0}=0-55$, and blade tip Reynolds numbers $\operatorname{Re}=2 \pi f R_{0} c / v=0-2 \times 10^{5}$, where $v$ is the kinematic viscosity of water. Since the free-stream velocity does not vary, the rotational speed is expressed by the dimensional frequency $f$ throughout the paper, for the sake of simplifying the presentation - it can easily be converted to the non-dimensional tip speed ratio, if needed. The blade pitch angle $\beta_{0}$ 


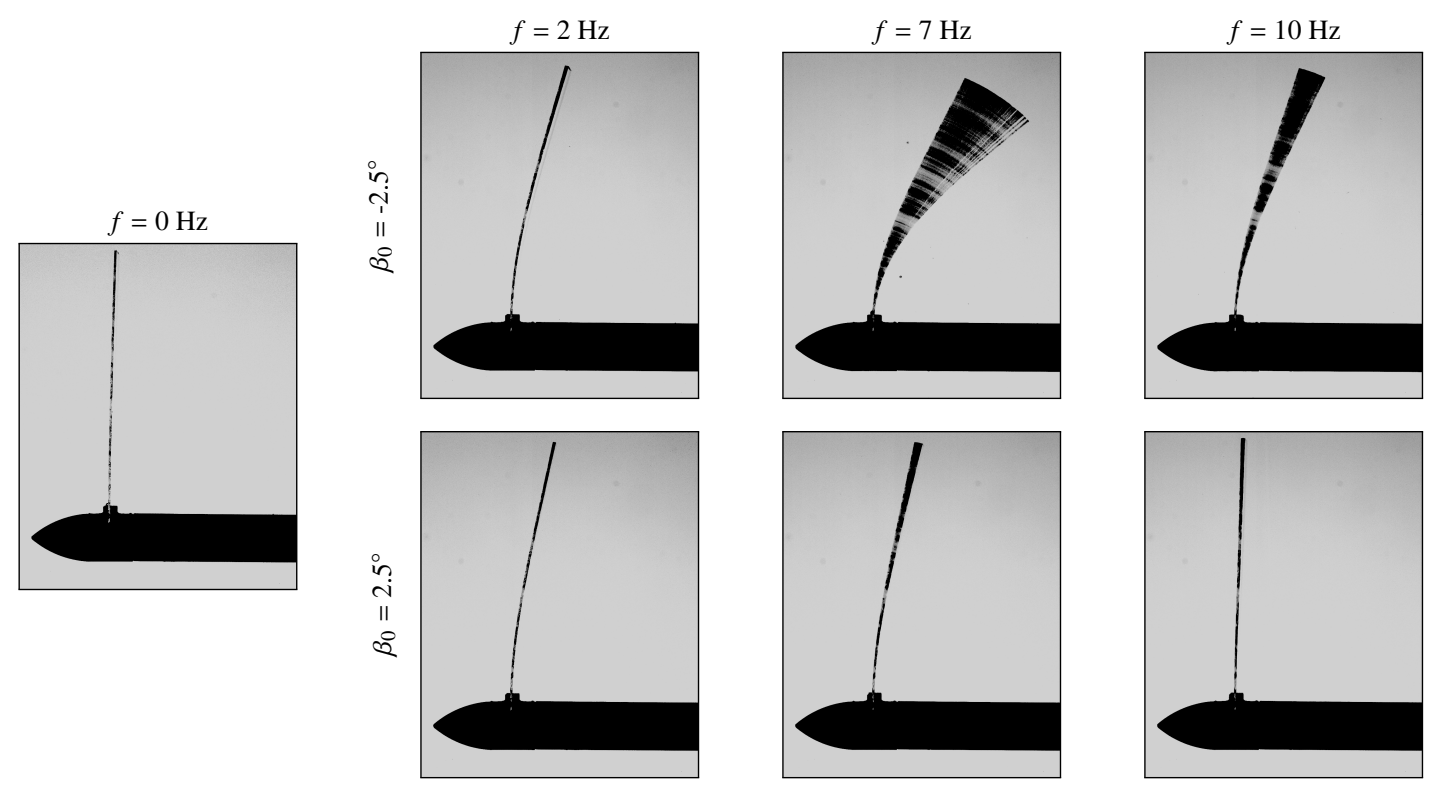

Fig. 5. Flapwise deformation of the blade with $\left|\beta_{0}\right|=2.5^{\circ}$, as function of rotational frequency. Superpositions of $50-100$ phase-locked images.

was varied in the range $-10^{\circ}<\beta_{0}<10^{\circ}$.

\section{Blade deformation}

\subsection{Flapwise bending}

In this section, the flapwise bending behaviour of the rotor blade is presented, showing the effect of blade pitch angle and rotational frequency on this type of deformation. First, the overall deformation behaviour, as determined from the visualizations, is described. At the end of the section, an analysis of the observations with some explanations is presented.

Figure 5 provides a survey of the observed bending for the blade with absolute pitch $\left|\beta_{0}\right|=2.5^{\circ}$, which is representative of a range of smaller pitch angles. Without rotation $(f=0)$, the blade is almost straight; it is very slightly bent, because of the non-zero free stream. As the rotation frequency increases, the blade deforms further in the downstream direction, for both positive and negative pitch angles, until a maximum deflection is reached for $f \approx 5-7 \mathrm{~Hz}$. Increasing $f$ further, the deformation decreases again towards the original unbent position. The images in Fig. 5 are superpositions of a large number of photos taken at the same phase of the rotor rotation. Whereas for positive pitch values, the blade position is very nearly constant in time, a certain spread is observed for negative pitch at higher frequencies. The amplitude of this fluctuation is greatest around $f=7 \mathrm{~Hz}$. It is caused by a low-frequency unsteadiness of the flow behind the rotor, which is analysed in more detail in section 5. The overall behaviour is similar for the blade with zero pitch $\left(\beta_{0}=0\right)$.

A different sequence of bending deformations is observed for blades pitch angles $\left|\beta_{0}\right|=5^{\circ}$ (Fig. 6) and higher. Increasing the rotation speed for negative pitch again leads to a downstream bending of the blade. However, contrary to the lower-pitch case, the bending continues to increase with $f$, resulting in extreme deformations, where the blade tip almost touches the rotor shaft. This increase continues even beyond the highest frequency shown in Fig. 6, until the blade eventually breaks at $f=14 \mathrm{~Hz}$. Unsteady fluctuations of the blade position are again observed for intermediate frequencies of rotation, with significantly higher amplitudes than for the low-pitch blades, and a maximum at $f=7.5$ $\mathrm{Hz}$.

For positive pitch $\left(\beta_{0}=5^{\circ}\right)$ and increasing rotor frequency, the blade again first bends in the downstream direction, reaches a maximum around $f=2 \mathrm{~Hz}$, after which the deformation decreases. This is initially similar to the lowpitch case in Fig. 5. However, the high-pitch blade continues to bend in the upstream direction, up to very large 

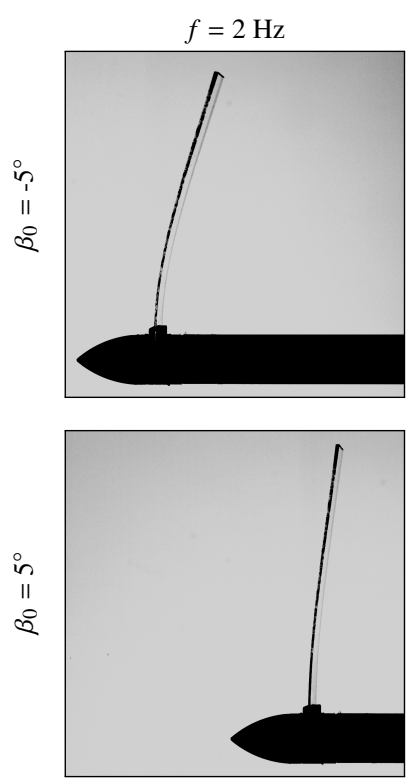
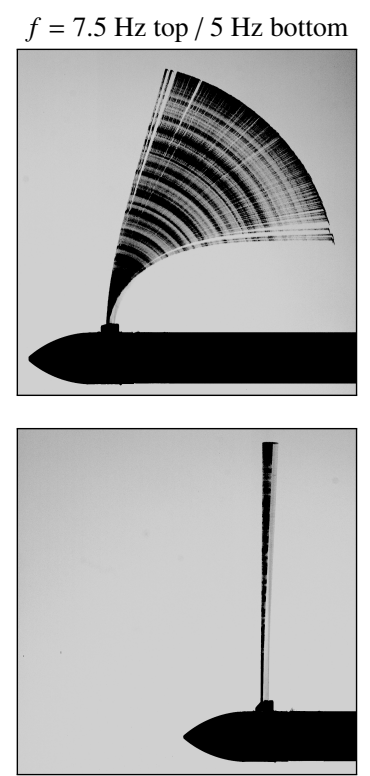

Fig. 6. Same as Fig. 5, for $\left|\beta_{0}\right|=5^{\circ}$.
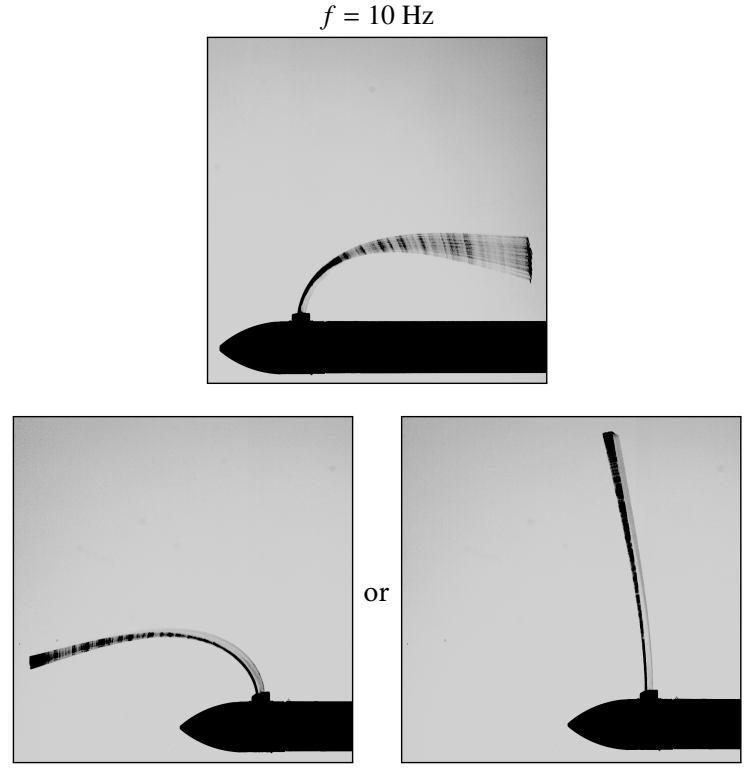

deformations, causing it to break for $f>10 \mathrm{~Hz}$. The same sequence is qualitatively found for blades with higher pitch angles $\left(\left|\beta_{0}\right|=7.5^{\circ}\right.$ and $\left.10^{\circ}\right)$.

An additional interesting feature was observed for the $\beta_{0}=5^{\circ}$ blade. When repeating the same experimental sequence of increasing the rotation frequency several times, a second deformation pattern appeared randomly at higher $f$. It is shown in Fig. 6 at $f=10 \mathrm{~Hz}$ (bottom right). Instead of the strong upstream bending mentioned above, the blade changes its deformation direction again and straightens out, which is similar to the behaviour of the low-pitch blades at high frequency. This suggest that the pitch of $\left|\beta_{0}\right|=5^{\circ}$ represents a limit between high- and low-pitch behaviour for the current rotor configuration.

For a more quantitative representation of the flapwise blade deformations and their fluctuations, an analysis method based on the blade-tip positions was devised. Figure 7 shows a superposition of all the blade images recorded for the $\left|\beta_{0}\right|=5^{\circ}$ blade. The dots are the tip positions delimiting the fluctuation intervals observed for each rotation frequency, such as the ones in Fig. 6. These positions all lie, to a good approximation, on a single circle, whose centre is shifted by $\sim 2 \mathrm{~cm}\left(\sim 22 \%\right.$ of $\left.R_{0}\right)$ in the radial direction from the rotor centre. The angular position $\theta$ of the blade tip on this circle is then used to characterise the magnitude of the flapwise (streamwise) deformation, with $\theta=0$ representing the undeformed blade. Figure 8 shows a summary of these measurements concerning the mean deflection and the fluctuation range, for the cases representing low pitch $\left(\left|\beta_{0}\right|=2.5^{\circ}\right)$ and high pitch $\left(\left|\beta_{0}\right|=5^{\circ}\right)$ of Figs. 5 and 6 , respectively.

Figure 9 contains the streamwise deformation data from all blades tested in this study. It shows again the two qualitative types of behaviour, depending on the blade pitch angle. For low pitch $\left(\left|\beta_{0}\right|<5^{\circ}\right)$, the bending increases with rotation frequency for both positive and negative pitch up to $f \approx 5 \mathrm{~Hz}$, and then decreases again towards zero. The fluctuation amplitude for negative pitch remains moderate, not exceeding $10^{\circ}$. For high pitch $\left(\left|\beta_{0}\right| \geq 5^{\circ}\right)$, the mean deformations do not decrease to zero at high rotation rate, but continue to increase to extreme values, bending the blade almost all the way to the rotor axis (downstream for negative pitch, upstream for positive pitch) until it breaks. The unsteady fluctuations for negative pitch are about three times larger than for the low-pitch blades.

In order to gain some understanding of the deformation pattern, one has to consider the fluid forces acting on the blade during rotation, which vary with the effective angle of attack of the rotor blade. This angle is a function of the pitch, the induced twist and the angle of the total flow in the rotor plane, as explained above (Fig. 2b). For a positive angle of attack, the lift force is directed upstream, which leads to a flapwise bending of the blade in the same direction, and vice versa for a negative angle of attack.

The blade pitch angle is a geometrical constant along the blade and is affected by neither the flow speed, the 


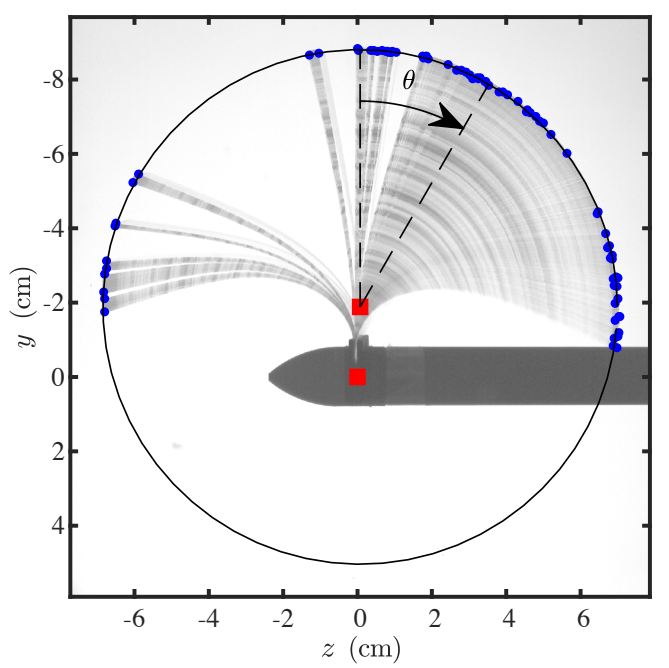

Fig. 7. Illustration of the method used to characterise the magnitude of the flapwise blade deformations.

(a)

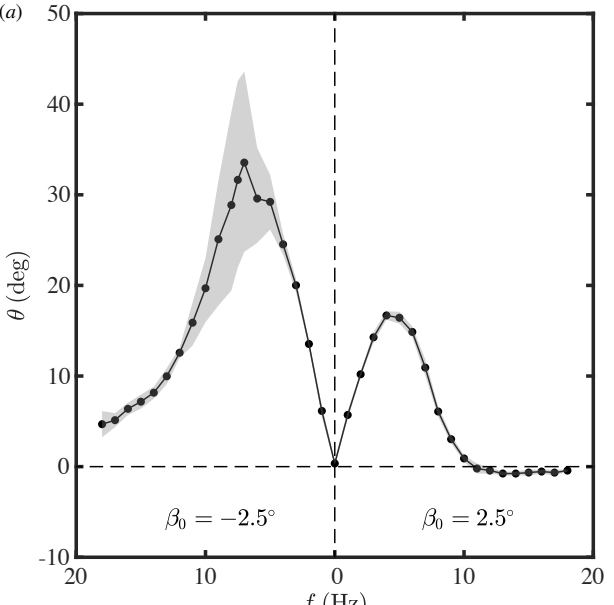

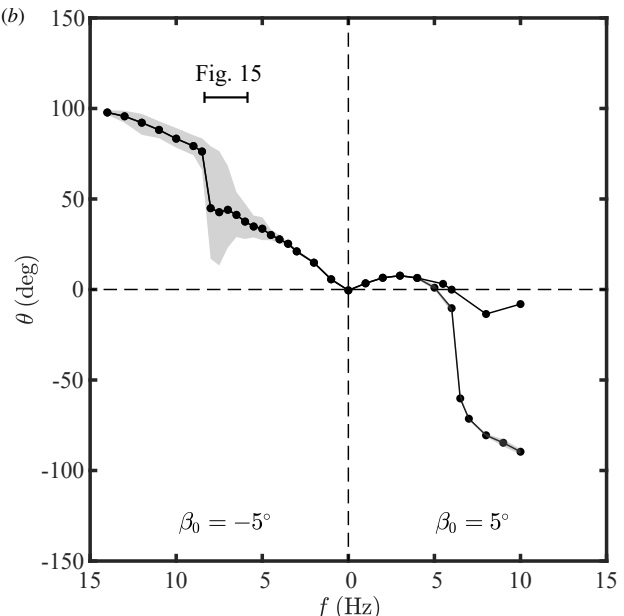

Fig. 8. Flapwise deformation as function of rotation frequency for blades with (a) $\left|\beta_{0}\right|=2.5^{\circ}$ and (b) $\left|\beta_{0}\right|=5^{\circ}$. The line and dots represent the mean deflection, and the grey area the fluctuation range.
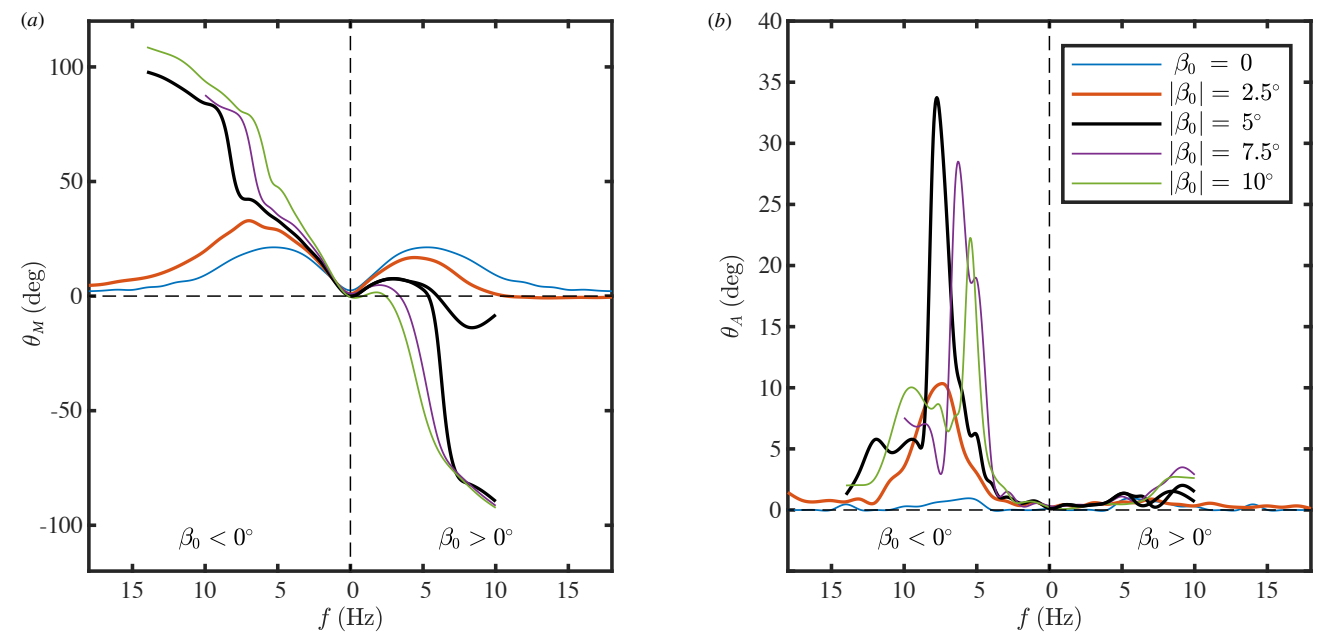

Fig. 9. (a) Mean deflection $\theta_{M}$ and (b) amplitude $\theta_{A}$ of the deformation fluctuation for all tested blades. 

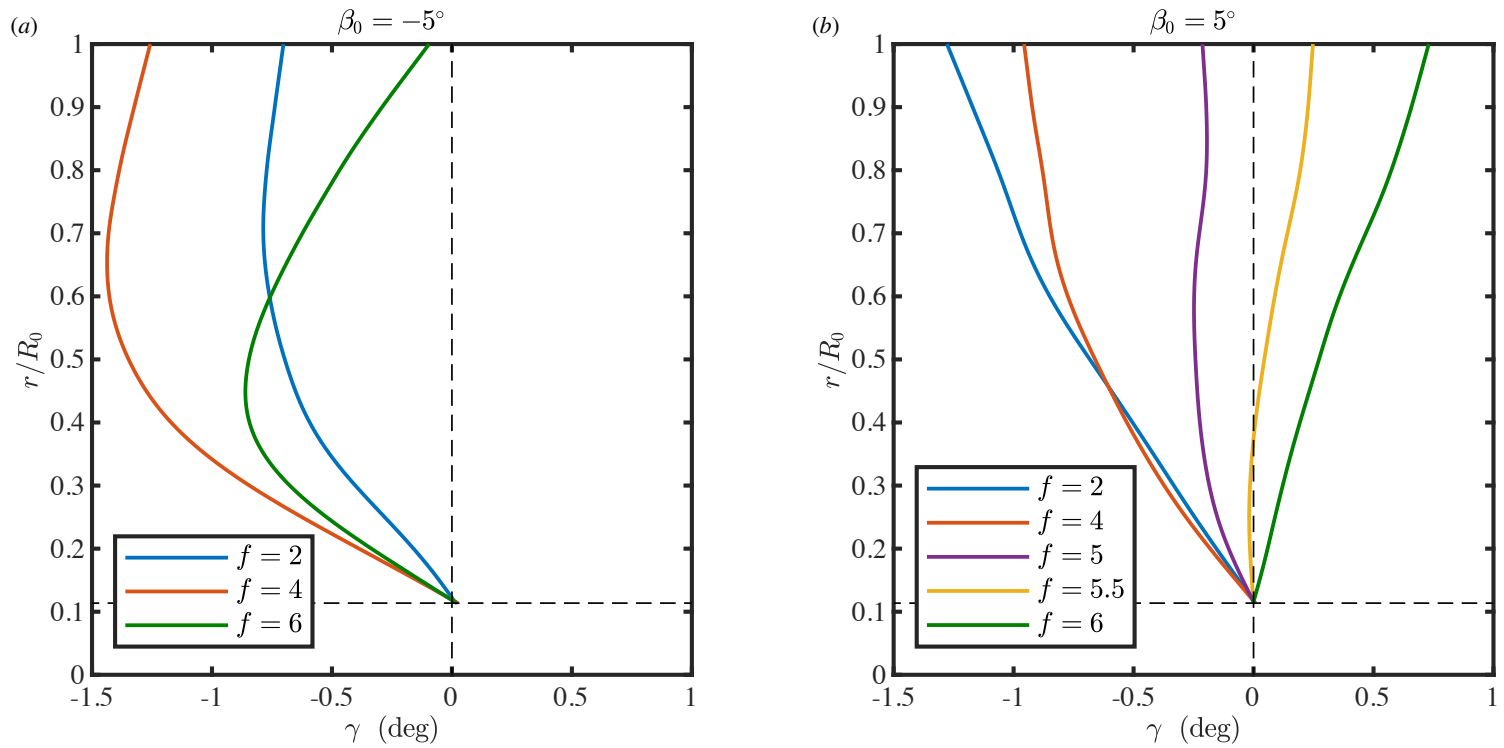

Fig. 10. Twist distributions along the rotor blade for (a) $\beta_{0}=-5^{\circ}$ and (b) $\beta_{0}=5^{\circ}$ at various rotation rates.

rotor rotational speed nor the flapwise bending. However, the flow angle varies along the blade, it depends on the free-stream velocity, the local rotation velocity, which is proportional to the distance from the axis, and the induced axial and azimuthal velocities of the rotor. As the tip speed ratio increases, the flow angle $\varphi$ decreases and eventually becomes negligible compared to the pitch and induced twist angles at the blade tip. If in addition the twist is small (see next section), this means that the effective angle of attack at high rotation frequency is mainly determined by the blade pitch. This explains the strong streamwise deformations observed for high-pitch blades. At high rotation, the angle of attack remains high, and the strong fluid forces, proportional to the square of the relative velocity, bend the blade. At low pitch, the fact that the blades straighten up again at high rotation indicates that the effective angle of attack must be close to zero, i.e. that the small pitch is compensated by the angle of the induced flow and/or the blade twist, which appears not to be possible at higher pitch. In the current set of experiments, the total axial velocity in the rotor plane near the tip is always positive (see section 4). This means that the contribution from the flow angle to the effective angle of attack is always negative there. For negative pitch, this enhances the magnitude of the angle of attack, while for positive pitch, the interplay between pitch, twist and flow angle can lead to a change of sign of the effective angle of attack, as the rotation rate is increased, resulting in a reversal of the deformation trend. This aspect is explored in more detail for the cases with small bending magnitude in section 4 below. For cases with stronger deformation, it was not possible to determine the effective angle of attack, since the three-dimensional geometry of the blade could not be measured with the current DIC set-up.

\subsection{Torsion}

Figure 10 contains the radial twist distributions of the blade with pitch $\left|\beta_{0}\right|=5^{\circ}$, in the accessible range of deformations, which corresponds to rotation frequencies up to $f=6 \mathrm{~Hz}$. For negative pitch (Fig. 10a), the blade exhibits a negative twist distribution along the span. The twist decreases from zero at the hub junction until it reaches a minimum value, after which it increases again up to the blade tip. The magnitude (absolute value) initially increases with the rotational speed reaching $|\gamma| \approx 1.45^{\circ}$ at about $2 / 3$ of the span and $|\gamma| \approx 1.3^{\circ}$ at the tip for a frequency of $f=4 \mathrm{~Hz}$. This trend changes for higher rotation rates. At $f=6 \mathrm{~Hz}$, the blade exhibits strong flapwise bending fluctuations (see Figs. 6 and 9b), which are accompanied by a time-dependent twist distribution. The result shown in Fig. 10(a) for $f=6 \mathrm{~Hz}$ is the time-averaged twist distribution. The magnitude of the minimum is reduced $\left(|\bar{\gamma}| \approx 0.8^{\circ}\right.$ at $r / R_{0} \approx 0.45$ ), and the mean twist almost vanishes at the blade tip.

A different behaviour is observed for positive pitch $\left(\beta_{0}=5^{\circ}\right.$, Fig. 10b). At a low rotational speed, the blade has a negative twist distribution, which is nearly linear along the span, with the minimum at the tip. As the frequency is 
increased, the twist profile remains approximately linear, but the the slope and the tip value change. Between $f=4 \mathrm{~Hz}$ and $f=6 \mathrm{~Hz}$, the tip twist evolves from $\gamma \approx-1^{\circ}$ to $\gamma \approx 0.7^{\circ}$, i.e. the twist changes its sign with increasing $f$. The twist influences the effective angle of attack of the blade. As shown in the next section, the twist evolution in Fig. 10(b) is accompanied by a change of sign of the angle of attack near the tip, and a corresponding change in the tip vortex circulation. A non-zero radial derivative of the twist angle at $r / R_{0}=1$ indicates that the blade experiences a twisting moment at the tip. This is particularly pronounced for $f=2 \mathrm{~Hz}$ and $f=6 \mathrm{~Hz}$ in Fig. 10(b). These are also the cases where the blade tip vortices are most pronounced (Fig. 12a,c below), suggesting that this moment is likely to be related to the presence and strength of this vortex. The role played by the twist will become clearer when comparing with the results obtained for a rigid blade (section 6.1).

\section{Flow field}

For a better understanding of the deformation behaviour of the flexible rotor, flow field measurements were made using Particle Image Velocimetry. Figures 11 and 12 present the results for high-pitch blades, which exhibit the largest flapwise deformations, as function of the rotation frequency, for negative and positive pitch, respectively. Shown are the phase-averaged and time-averaged azimuthal vorticity, as well as the total axial velocity component, in the plane containing the rotor axis. In addition, radial profiles of the non-dimensional time-averaged induced axial velocity $u_{i} / U_{0}=\left(U-U_{0}\right) / U_{0}$, extracted from the PIV data at various downstream positions $(z)$, are plotted in Fig. 13.

For negative pitch $\left(\beta_{0}=-5^{\circ}\right)$ and low rotation rate $(f=2 \mathrm{~Hz})$, tip and root vortices can be clearly identified (Fig. 11a). The rotor-induced velocity is directed upstream, but its value is moderate at this low frequency (Fig. 13a). The lower axial velocity behind the rotor causes the wake to expand radially, due to volume conservation. As the rotation rate is increased, the induced velocity gradually exceeds the free-stream velocity (Fig. 13b-e), leading to a significant wake expansion and the formation of a large-scale recirculation zone behind the rotor. This regime is accompanied by the strong bending fluctuations discussed in the previous section. Due to the unsteadiness of the recirculation zone, the phase- and time-averaged vorticity fields in Fig 11c-e are very similar. Unsteady recirculation in the flow around a rotor is reminiscent of the so-called Vortex Ring State (VRS), which occurs for helicopters in steep descent (Leishman, 2006). This aspect is analysed in more detail in section 5. A qualitatively similar general flow pattern is found for all blades with negative pitch. As the rotational frequency reaches higher values, the low-pitch blades straighten out again, whereas the high-pitch blades become more and more deformed, with the recirculation region decreasing in size and strengthening (Figs. 11e and 13e).

For positive pitch angles, (Figs. 12 and 13f-j), the induced axial velocities are generally lower. At low frequency $(f=2 \mathrm{~Hz}$ ), the flow resembles the negative-pitch case, with tip and root vortices in the rotor wake, as well as an additional intermediate vortex indicating a sharp change of bound circulation along the blade span. For higher rotation rates, the negative axial induction near the blade tip decreases in magnitude, and eventually changes sign, becoming increasingly positive for $f \geq 6 \mathrm{~Hz}$ (Fig. 13h-j). This is reflected by the change of sign in the tip vortex vorticity seen in Fig. 12, and by the increasing upstream bending of the blade. The lift force is now directed upstream near the tip, causing a significant bending moment there. When the total axial induction, calculated over the entire rotor disk, is positive, volume conservation causes a radial contraction of the wake (Figs. 12d and 13i). For the case of extreme upstream deformation of the blade with $\beta_{0}=7.5^{\circ}$ at $f=8 \mathrm{~Hz}$, very strong downstream induction is found in front of the rotor, in the region enclosed by the strongly bent blade (Figs. 12e and 13j). Recirculation is also found for negative pitch, near the rotor axis, as seen in the axial velocity fields in Fig. 12. The streamwise extent of these zones reduces with increasing rotation rate.

The change of sign of the tip vortex circulation is also observed for the blades with pitch angles $\beta_{0}=2.5^{\circ}$ and $7.5^{\circ}$. The rotation frequency at which this change occurs decreases with increasing pitch angle. The tip vortex circulation is given by the blade's bound circulation in the tip region, which in turn depends on the effective angle of attack. This angle $(\alpha)$ was determined using Eq. (1) for the first three cases in Fig 12, where the tip vortex reversal occurs. The pitch $\beta_{0}$ is known and constant, and the twist $\gamma$ was measured using the DIC technique (Fig. 10b). The flow angle $\varphi$ was calculated from the approximate relation $\varphi=\tan ^{-1}[U /(2 \pi r f)]$, where the induced azimuthal velocity is neglected. $U$ is the time-averaged axial flow velocity obtained from PIV, which should in principle be evaluated in the rotor plane. Since the presence of the blade at this location in the PIV images perturbed the velocity measurements, profiles of $U$ extracted a short distance $\left(\sim 5 \%\right.$ of $\left.R_{0}\right)$ upstream of the rotor plane were used. The results are plotted in 

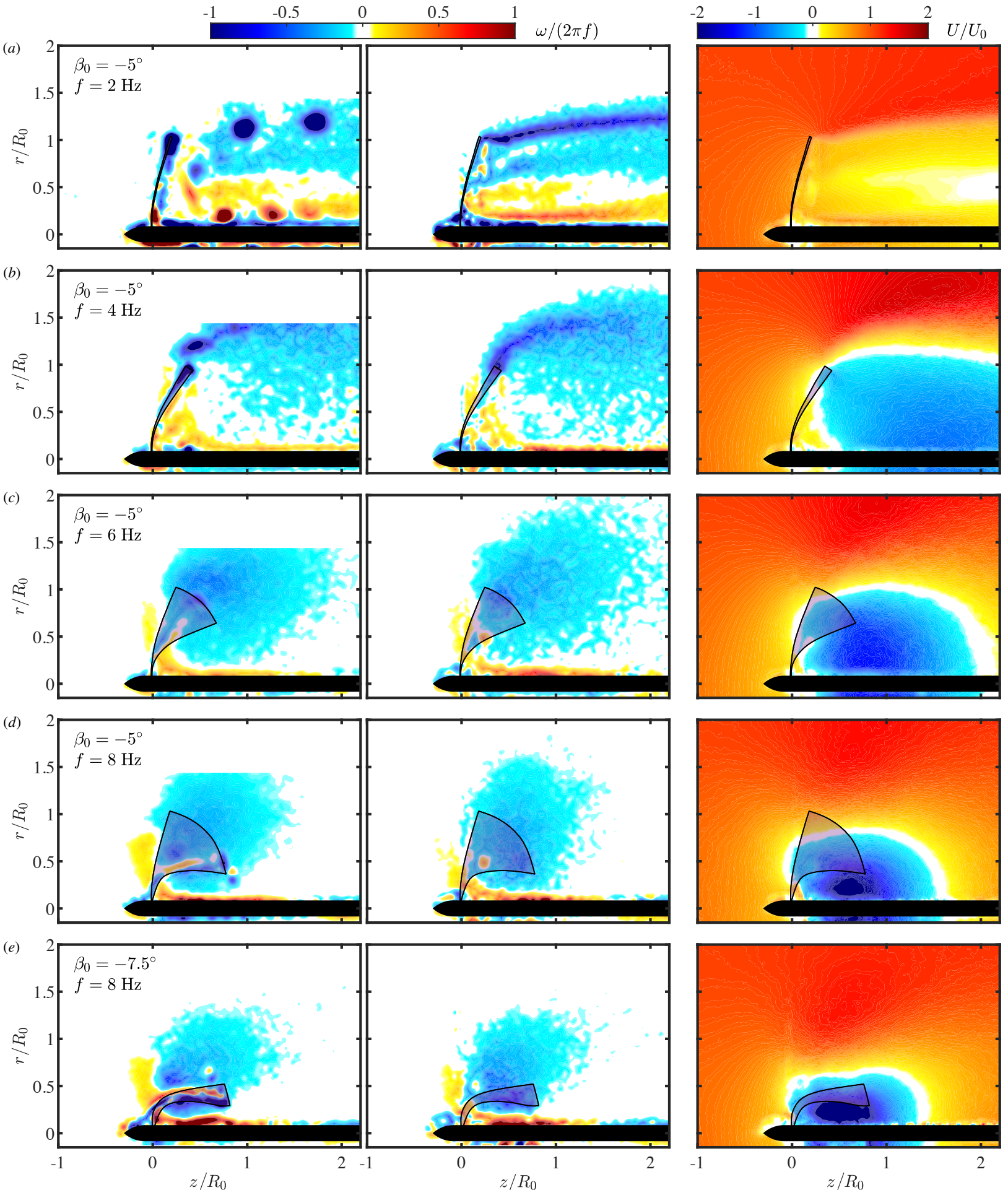

Fig. 11. Flow field measurements in the centre plane of the rotor wake, for blades with high negative pitch at various rotation rates. (Left) Phaseaveraged azimuthal vorticity. (Middle) Time-averaged vorticity. (Right) Time-averaged axial velocity. The shaded areas indicate the range of blade positions for each case. 

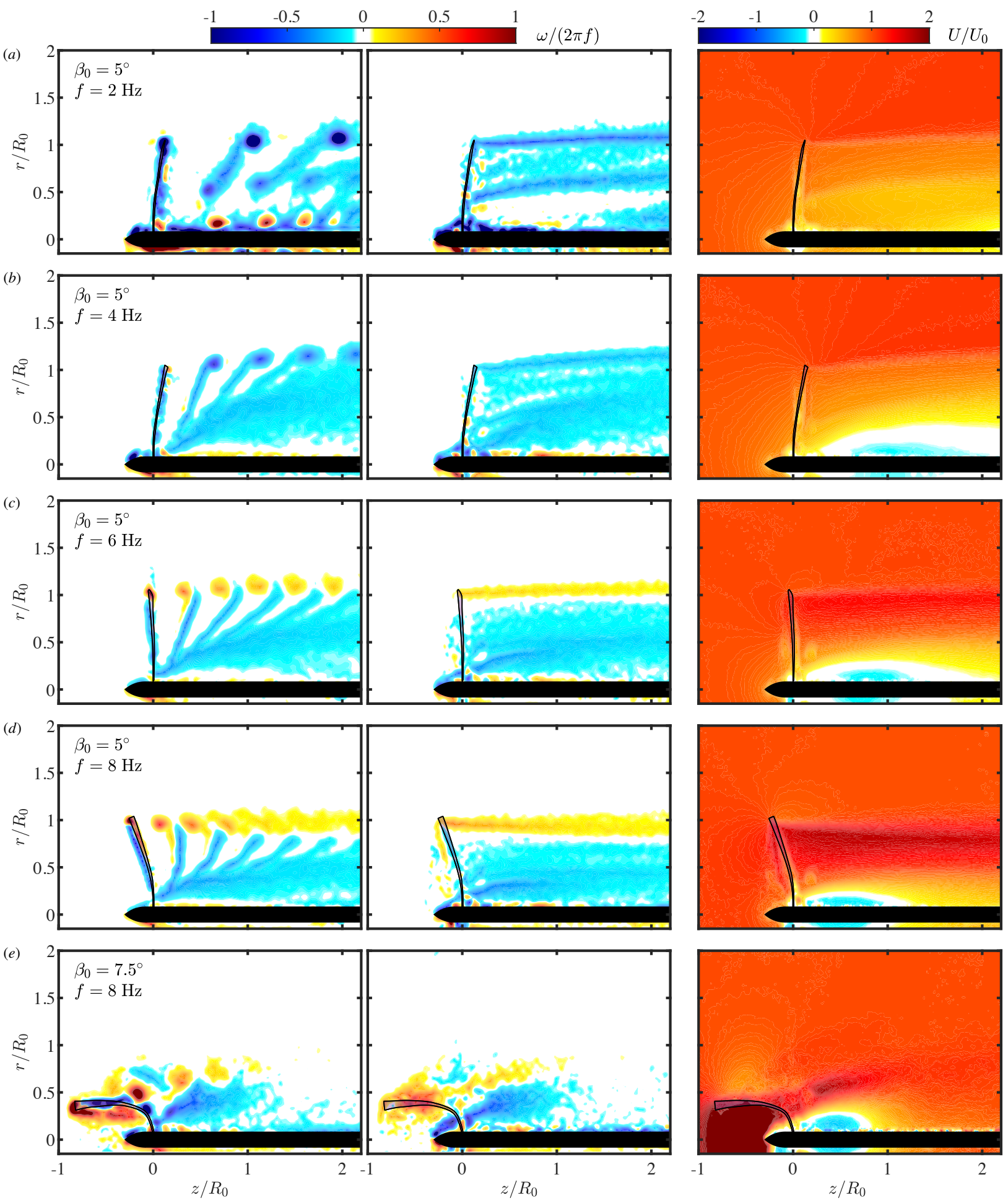

Fig. 12. Same as Fig. 11, for blades with high positive pitch. 

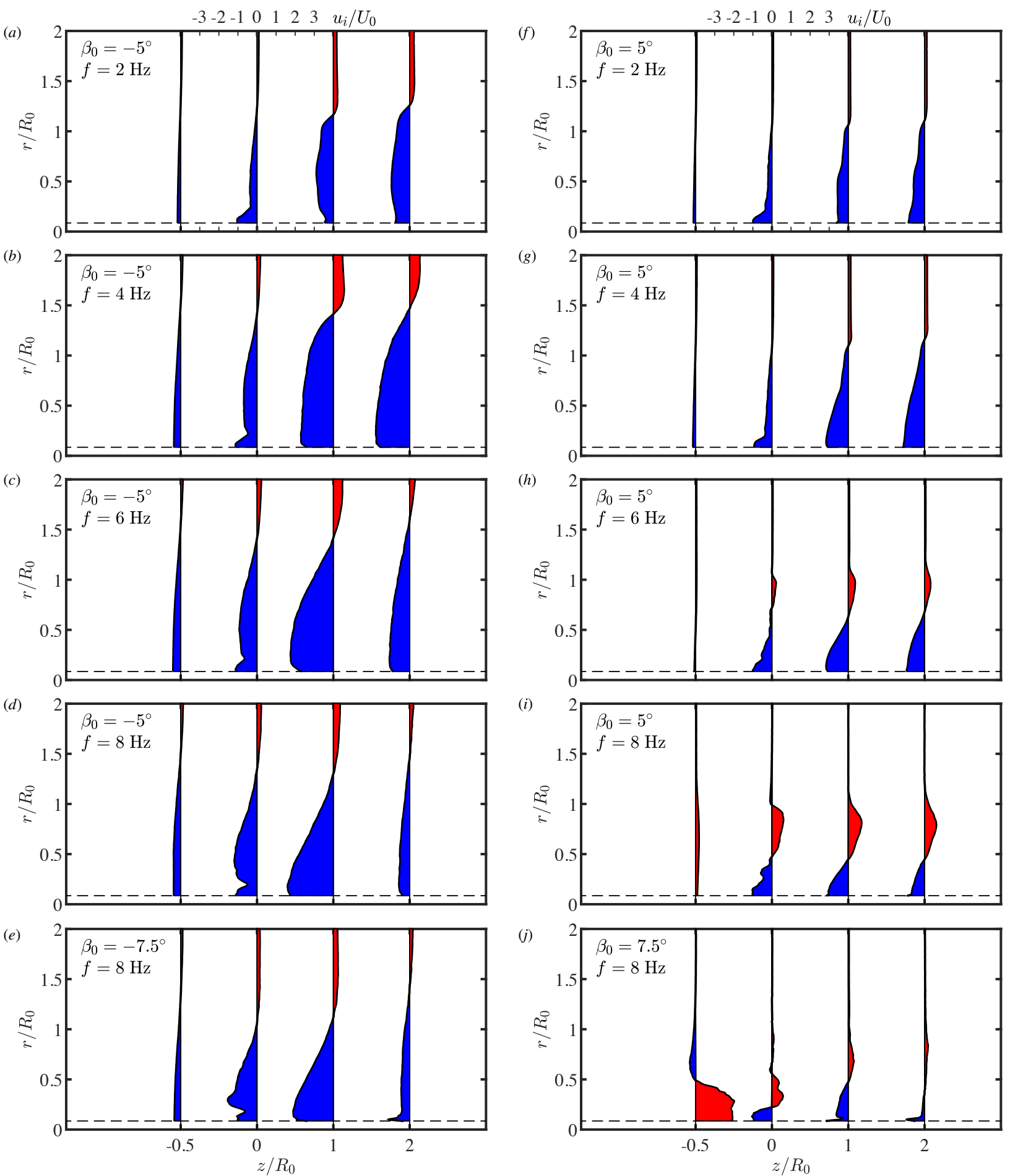

Fig. 13. Radial profiles of the time-averaged induced axial velocity at different downstream positions, for various rotation rates. Blue indicates upstream induction, red downstream induction. (Left) $\beta_{0}=-5^{\circ}$. (Right) $\beta_{0}=5^{\circ}$. 

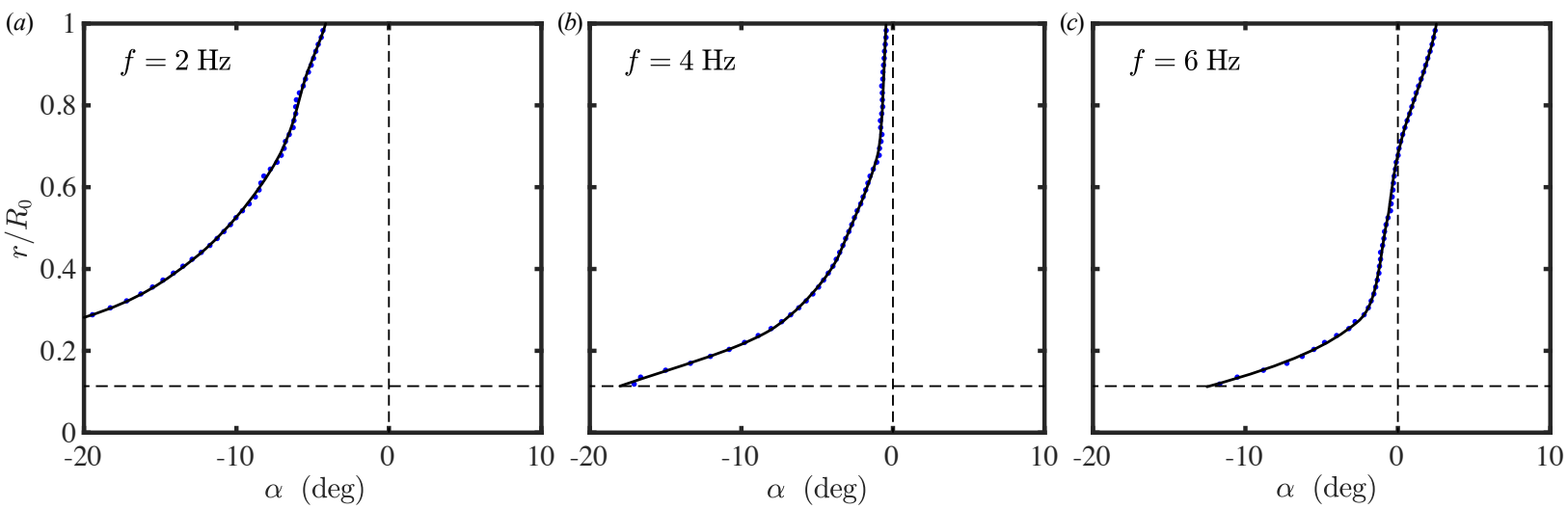

Fig. 14. Radial profiles of the effective angle of attack $\alpha$ (see Fig. 2), for the blade with pitch $\beta_{0}=5^{\circ}$. (a) $f=2 \mathrm{~Hz}$. (b) $f=4 \mathrm{~Hz}$. (c) $f=6 \mathrm{~Hz}$.

Fig. 14. They confirm that, for the blade with pitch $\beta_{0}=5^{\circ}$, the effective angle of attack changes sign between $f=4$ $\mathrm{Hz}$ and $f=6 \mathrm{~Hz}$ in the region near the tip.

\section{Unsteady blade deformation}

In the following, we analyse in more detail the unsteady bending observed for blades with negative pitch. Fig. 15 shows time histories of the bending magnitude $\theta$ (see Fig. 7) for the $\beta_{0}=-5^{\circ}$ blade in the frequency interval $6 \mathrm{~Hz} \leq f \leq 8.5 \mathrm{~Hz}$, over which large fluctuations are found (see Fig. 9b). For these measurements, $\theta$ was determined from series of phase-locked PIV image pairs (so that the corresponding flow field could be obtained simultaneously), recorded over a time period of $600 \mathrm{~s}$, which represents 3600 to 4800 rotor revolutions (only $300 \mathrm{~s}$ intervals are plotted in Fig. 15, for better clarity). The acquisition frequency was limited to about $2 \mathrm{~Hz}$, i.e. an image pair was recorded every 3 or 4 blade revolutions. This turned out to be sufficient to resolve the fluctuations of the bending magnitude, which occurred at very low frequency.

At the beginning of the unsteady bending regime, at $f=6 \mathrm{~Hz}$, the mean bending magnitude is near $\theta_{M}=40^{\circ}$, and the fluctuations around this value are small, of the order of $\pm 10^{\circ}$. At $f=7 \mathrm{~Hz}$, the mean bending has increased slightly, but the fluctuation interval around this mean has roughly doubled. The probability density function (PDF) of the bending magnitude, shown in the right column of Fig. 15 for each case, still shows a symmetric distribution around the mean. This changes at a slightly higher frequency $(f=7.5 \mathrm{~Hz})$. From the PDF in Fig. 15c, it appears as if the previous peak broadens even more, but at the same time a new narrower peak appears in the distribution, centred around $\theta=70^{\circ}$. At $f=8 \mathrm{~Hz}$, this new peak has become dominant, with only a few excursions to lower values of the bending magnitude remaining. Finally, at $f=8 \mathrm{~Hz}$, the fluctuations strongly reduce in amplitude again, with a mean bending magnitude close to $80^{\circ}$.

This sequence appears to represent a gradual shift between two discontinuous branches of the bending magnitude, as the frequency of rotation is increased. The two branches can actually be identified on the left side of Fig. 9b. During the transition, for $f=7.5$ and $8 \mathrm{~Hz}$, the rotor wake seems to intermittently switch between the states associated with the two amplitude branches, i.e. it exhibits a somewhat bistable behaviour in time. Bistability has been found previously in the turbulent wakes of three-dimensional bluff bodies, which are often inspired by the geometry of road vehicles (e.g., Lawson et al., 2007; Herry et al., 2011; Grandemange et al., 2013; Meile et al., 2016). A similarity with the present configuration may arise from the fact that this behaviour occurs for cases where the rotor generates a strong upstream induction, which produceds a recirculation region roughly comparable to the wake of a bluff body. In the current study, the additional ingredient of fluid-structure interaction with a rotating blade is present.

In order to further explore the idea of bistability, we analyse the time-dependent velocity field using a procedure proposed by Grandemange et al. (2013). The sequence of PIV fields corresponding to the bending time history in Fig. $15 \mathrm{~d}(f=8 \mathrm{~Hz})$ was conditionally averaged, once for $\theta \geq 60^{\circ}$, and once for $\theta \leq 40^{\circ}$, with the aim of determining the flow structure corresponding to the two intermittent states. The resulting vorticity distributions are shown in Fig. 16. The state with high bending of the blade, in which the rotor remains for most of the time, involves a strong 

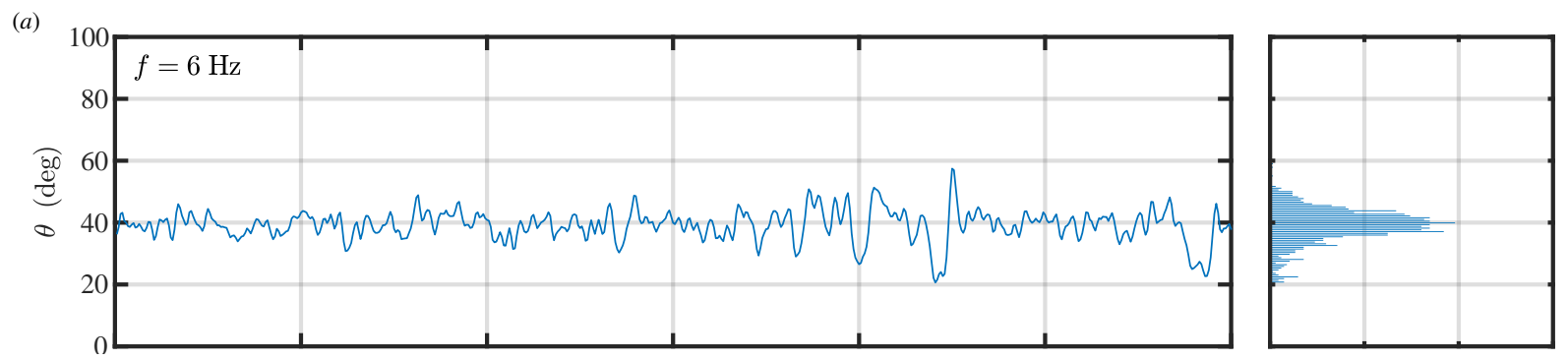

(b)
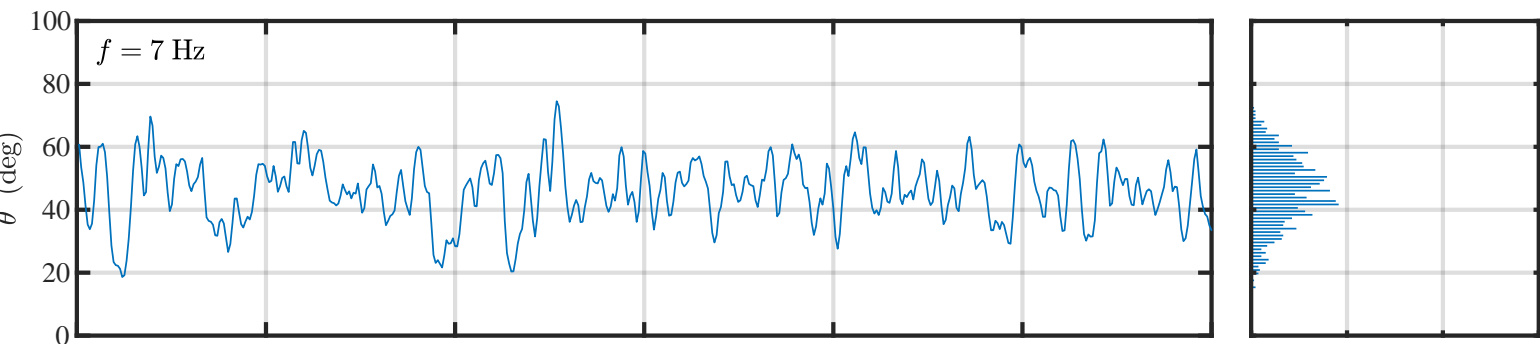

(c)
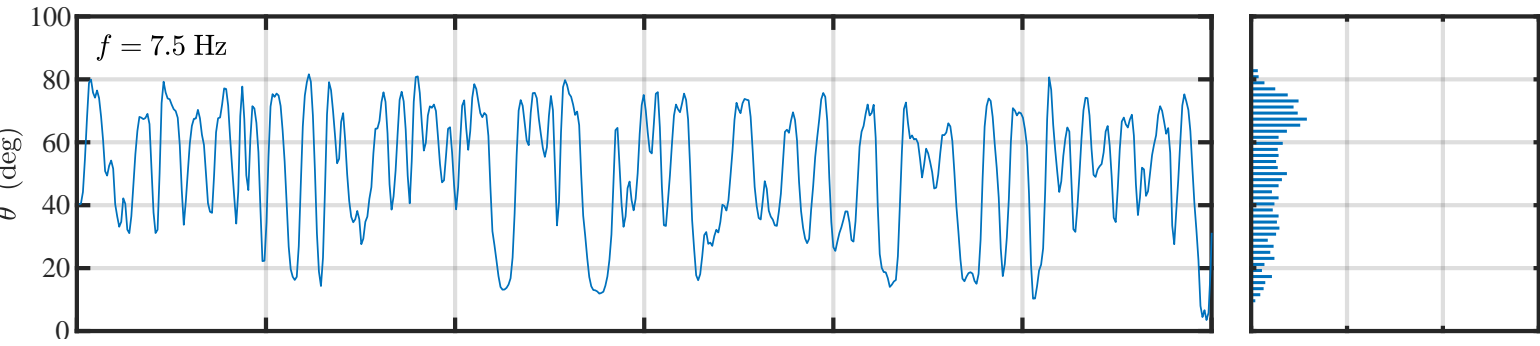

(d)
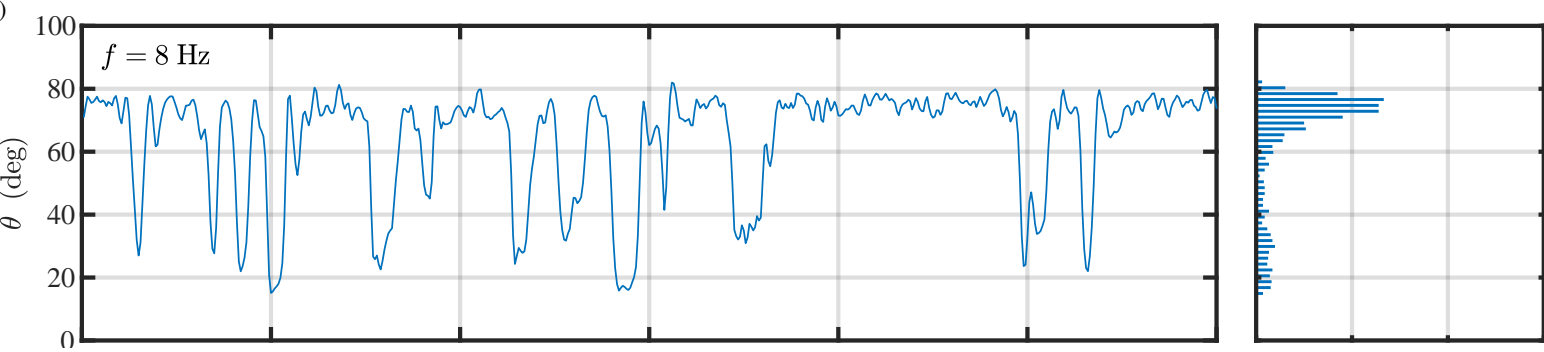

$(e)$

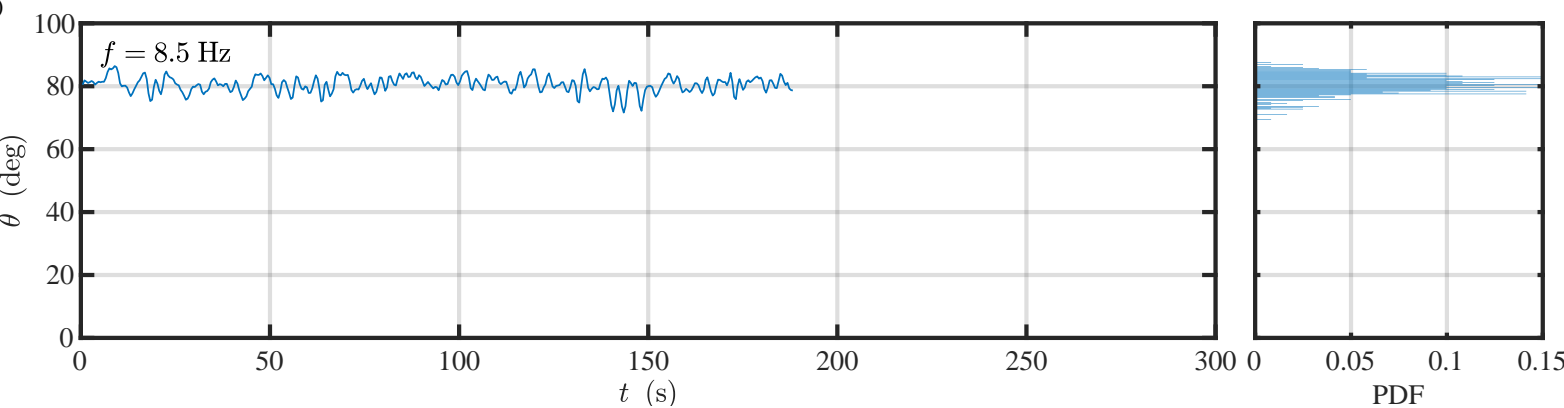

Fig. 15. (Left) Time history of the flapwise bending magnitude for the blade with pitch $\beta_{0}=-5^{\circ}$, for various rotation rates spanning the interval marked in Fig. 8(b). (Right) Corresponding probability density functions (PDFs). 

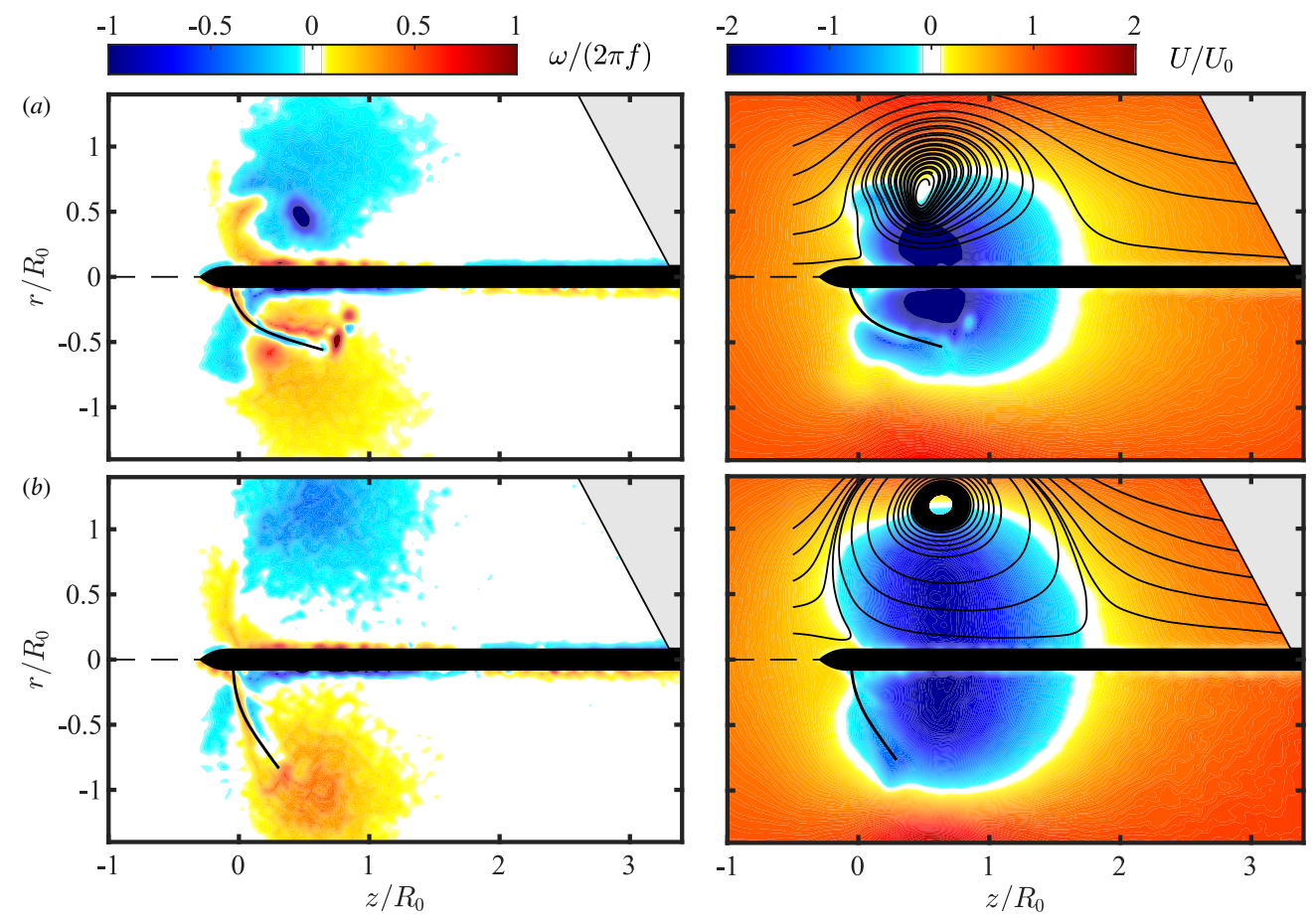

Fig. 16. Phase-averaged flow fields of the two states observed for the blade with pitch $\beta_{0}=-5^{\circ}$ rotating at $f=8 \mathrm{~Hz}$. (a) Average for $\theta>60^{\circ}$, (b) average for $\theta<40^{\circ}$. (Left) Azimuthal vorticity, (right) axial velocity with streamlines.

and concentrated recirculation zone surrounding the blade. This again resembles the flow structure of the helicopter Vortex Ring State. The state with moderate bending (Fig. 16b) occurs only intermittently. Its structure also exhibits recirculation and a vortex ring-like structure, which is less concentrated and extends further out in the radial direction. The Vortex Ring State arises from a merging of the blade tip vortices into a single large-scale structure, which remains near the rotor plane, instead of being swept downstream. Previous studies (e.g., Stack et al., 2005; Quaranta, 2017) have shown that this phenomenon is unsteady: the vortex ring is shed into the wake at low frequency (compared to the rotor frequency), which leads to strong variations in the fluid forces acting on the blades. The intermittent state in Fig. 16(b) may be related to such shedding events.

Time series as in Fig. 15 can be used to estimate the characteristic frequency $f_{b}$ of the bending deformations and accompanying wake structure. We focus on the case with $f=7.5 \mathrm{~Hz}$ (Fig. 15c), since it has the largest fluctuation amplitude (see Fig. 6). The frequency was calculated by counting the number of peaks of the time signal in the measurement interval, leading to an estimate of $f_{b}=0.15 \mathrm{~Hz}$, which is 50 times smaller than the blade rotation frequency. For the purpose of comparing with previous related studies, the frequency can be non-dimensionalized in the form of a Strouhal number ( $\mathrm{St}$ ), based on the rotor diameter and the free-stream velocity. When using the diameter of the undeformed rotor, one obtains $\mathrm{St}=2 f_{b} R_{0} / U_{0}=0.15$. However, it seems more appropriate to use the diameter $R^{*}$ of the deformed rotor, since it is this length that determines the size of the recirculation zone. With $R^{*} \approx 0.7 R_{0}$ on average, this leads to an effective Strouhal number $\mathrm{St}^{*}=2 f_{b} R^{*} / U_{0}=0.10$. The Reynolds number based on the same length and velocity scales is of the order of $10^{4}$. Stack et al. (2005) and Quaranta (2017) have performed experiments on rotor flows, including the Vortex Ring State. The former observe low-frequency thrust oscillations in this regime, whose period is 10 to 60 times longer than the rotor frequency, depending on the flow conditions, which is consistent with the present observations. Quaranta (2017) measured a Strouhal number of St $=0.17$ for wake fluctuations of an unyawed rigid one-bladed rotor in VRS. In a study of a two-bladed rotor wake in the wind turbine regime (weaker upstream induction without recirculation), Medici and Alfredsson (2006) also observed slow oscillations (wake meandering), with associated Strouhal numbers in the range $0.12<\mathrm{St}<0.2$, for the cases with the highest axial induction. Since rotors in the VRS and wind turbine regimes generate a velocity deficit in their wake, it has been suggested that the low-frequency oscillations may be related to vortex shedding behind bluff bodies. The 
most relevant bluff body geometry for comparison is the solid disk. Indeed, (Calvert, 1967) measured St $=0.14$ for a solid disk at Reynolds numbers between $10^{4}$ and $10^{5}$.

From these comparisons, we concluded that the low-frequency, large-amplitude bending fluctuations observed in our experiments with flexible blades of negative pitch are related to the dynamics of the wake behind the rotor. In the range of rotation and flow parameters where the fluctuations occur, the wake contains a large recirculation zone in the form of a vortex ring, which is periodically released into the flow, similarly to vortex shedding behind an axisymmetric body (disk). It should be noted that these fluctuations are very different from the high-frequency, low-amplitude flutter phenomenon, another commonly observed fluid-structure interaction involving lifting surfaces.

\section{Comparison with a rigid rotor}

The previous sections have shown that blade flexibility can lead to significant modifications of the geometry and the flow around a rotor operating in a dense medium like water. These arise mainly because of the large flapwise bending deformations that the blade undergoes, changing the effective radius of the rotor, which can also be timedependent when the blade responds to unsteadiness in the wake. One might ask what the effect of flexibility is in cases when the deformation remains small to moderate, i.e., when the overall geometry and flow structure is preserved. We tried to address this question by performing a set of PIV measurements for a rotor with the same dimensions as before, but equipped with a rigid blade made of stainless steel. We focus on configurations with large positive pitch $\left(\beta_{0}=5^{\circ}\right)$ and moderate rotation rate $(f \leq 6 \mathrm{~Hz})$, for which the bending magnitude remains small (see Fig. 8b). Higher frequencies could also not be reached, since the centrifugal forces acting on the single heavy metal blade cause a strong radial strain on the shaft, which leads to vibrations and mechanical instability.

\subsection{Wake structure}

Figure 17 shows the distributions of vorticity and axial velocity in the centre plane of the rigid rotor, for various rotation rates at positive pitch $\left(\beta_{0}=5^{\circ}\right)$, as well as one case for negative pitch $\left(\beta_{0}=-5^{\circ}\right)$ at $f=6 \mathrm{~Hz}$, which is where the regime of strong bending fluctuations sets in for the flexible blade. The radial profiles of the mean rotor-induced axial velocity for these cases are plotted in Fig. 18. These results can be compared to the corresponding ones for the flexible blade in Figs. 12(a-c), 11(c) and 13(f-h,c).

Overall, the vorticity and velocity fields around the rigid blade are very similar to the flexible-rotor case. At low frequency $(f=2 \mathrm{~Hz})$, the tip and root vortices, as well as the intermediate vortex, are again clearly visible. For increasing rotation rate, a narrow recirculation zone also appears near the rotor axis, and the tip vortex circulation changes sign, but at a lower value than for the flexible blade. At $f=4 \mathrm{~Hz}$ (Fig. 17b), on can already detect the opposite-signed vorticity along the rotor wake edge in the phase-averaged fields. At $f=6 \mathrm{~Hz}$, the flow behind the rigid rotor (Fig. 17c) is practically identical to the one behind the flexible one (Fig. 12c). The same similarities are found in the induced velocity profiles (Fig. 18 compared with Fig. 13).

The different frequency for the tip vortex reversal of the rigid blade can be explained by the absence of blade twist, which modified the effective angle of attack of the flexible blade. This angle was also calculated for the rigid rotor, using the same procedure as described in section 4, with $\gamma=0$. The results are compared in Fig. 19. In both cases, the angles increase with increasing rotation rate. The differences are of the order of the twist measured for the flexible blade (Fig. 10b), with the corresponding sign. At $f=4 \mathrm{~Hz}$, the twist is negative, decreasing the slightly positive angle of attack near the blade tip measured for the rigid rotor to negative values.

Unsurprisingly, significant differences in the flow field are found for cases where the flexible blade deformation is large and unsteady, such as for $\beta_{0}=-5^{\circ}$ and $f=6 \mathrm{~Hz}$ (Fig. 17d compared with Fig. 11c). The wake of the rigid rotor also exhibits a recirculation zone, of a much larger radial and streamwise extent, which resembles less the Vortex Ring State structure. The similarity between phase-averaged and time-averaged vorticity fields in Fig. 18(d) suggests again a more complex unsteadiness of the wake, but this aspect was not investigated further here.

\subsection{Thrust}

Another interesting aspect concerning flexible rotors is the question of how the flexibility influences the rotor performance. One way of evaluating this effect is to compare the thrust produced by a rigid and a flexible rotor having 

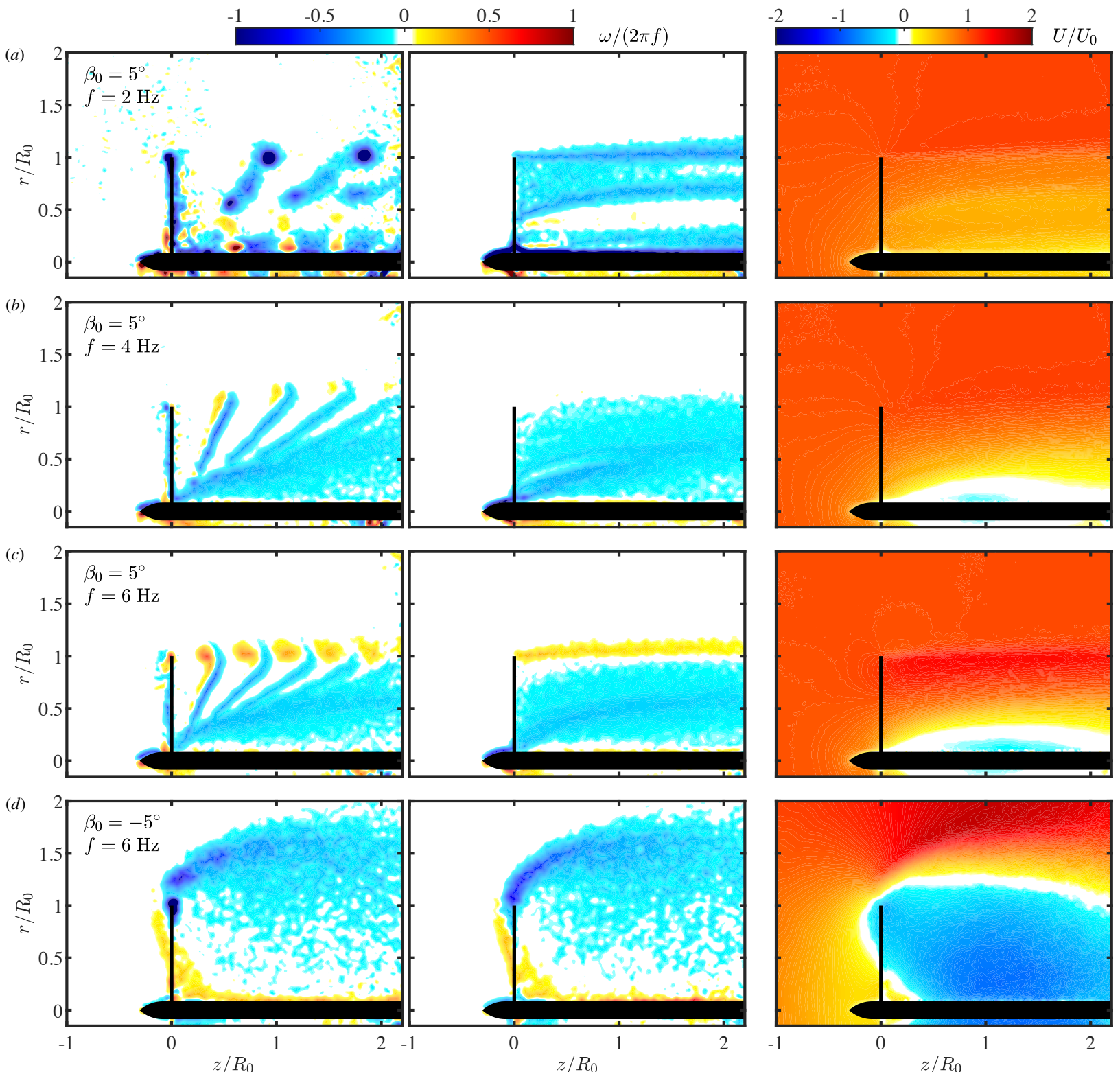

Fig. 17. Flow field in the centre plane of the rotor wake, for a rigid blade. (a) $\beta_{0}=5^{\circ}$ and $f=4 \mathrm{~Hz}$. (b) $\beta_{0}=5^{\circ}$ and $f=6 \mathrm{~Hz}$. (c) $\beta_{0}=-5^{\circ}$ and $f=6 \mathrm{~Hz}$. (Left) Phase-averaged azimuthal vorticity. (Middle) Time-averaged vorticity. (Right) Time-averaged axial velocity. 
(a)

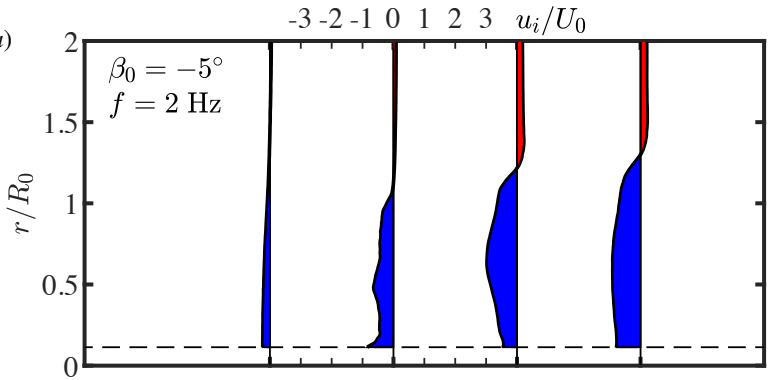

(b)

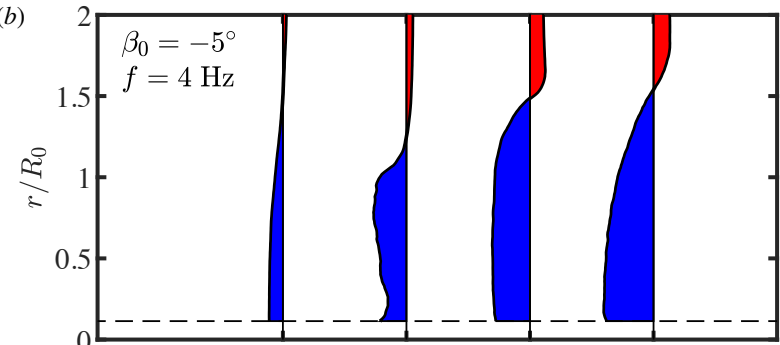

(c)

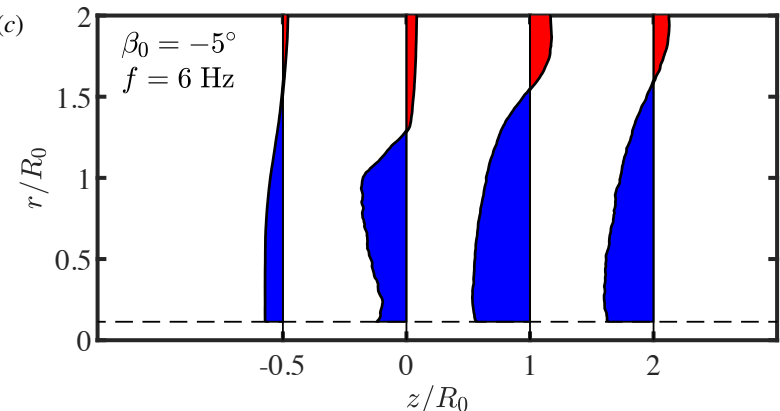

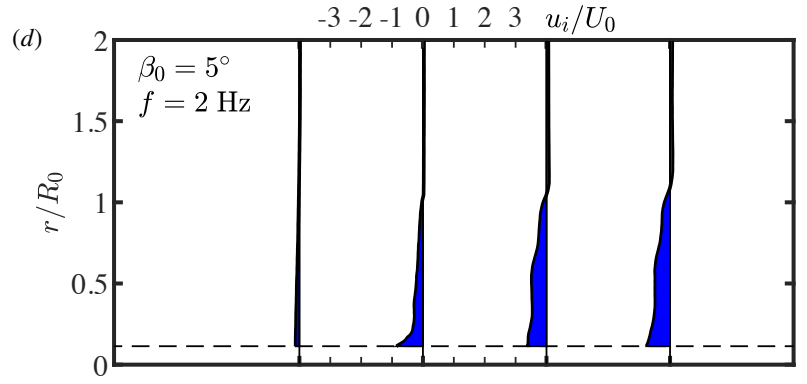

(e)
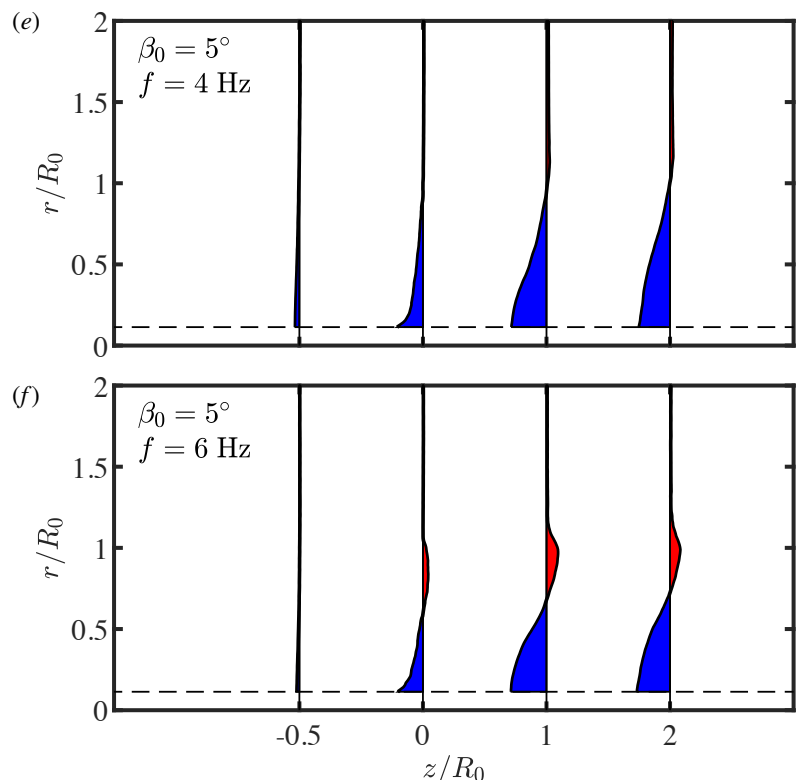

Fig. 18. Radial profiles of the time-averaged induced axial velocity at different downstream positions, for the rigid blade at various rotation rates. Blue indicates upstream induction, red downstream induction. (Left) $\beta_{0}=-5^{\circ}$. (Right) $\beta_{0}=5^{\circ}$.

the same geometry at rest. This was carried out for a number of cases where the flexible blade is still fairly straight, and the difference in the geometry is mainly due to the twist.

Since the experimental set-up was not equipped to perform force measurements, the rotor thrust was estimated from the mean axial velocity field, by calculating the rate of momentum change of the fluid flowing through the rotor, by considering a wake cross section far downstream. In practice, profiles of the average streamwise velocity $U(r)$ obtained by PIV at the position $z=2 R_{0}$ (Fig. 20a) were used to calculate this momentum change, i.e. the thrust $T$, as

$$
T=2 \pi \rho \int_{0}^{r_{\max }} U\left(U-U_{0}\right) r \mathrm{~d} r
$$

where $\rho$ is the water density. This method is (at least approximately) valid since the streamwise velocity is always positive in this cross section for the cases considered, and all streamlines originate in the upstream region where the axial velocity is equal to the free-stream velocity $U_{0}$. The integration is stopped at a radius $r_{\max }$, where the axial velocity reaches the value of the outer flow. Due to blockage effects in the water channel, this velocity is generally slightly larger than $U_{0}$, so that a further integration would lead to an erroneous overestimate of the thrust. The nondimensional thrust coefficient $C_{T}$ is calculated in the standard way, using the blade tip speed and the rotor area:

$$
C_{T}=\frac{T}{\frac{1}{2} \rho\left(2 \pi f R_{0}\right)^{2}\left(\pi R_{0}^{2}\right)}=\frac{T}{2 \pi^{3} \rho f^{2} R_{0}^{4}} .
$$

Figure 20(b,c) shows the thrust coefficients obtained in this way as function of rotation rate, for rotors with rigid and flexible blades and for two values of positive pitch. For both pitches, the rotor has negative thrust at low frequency, 

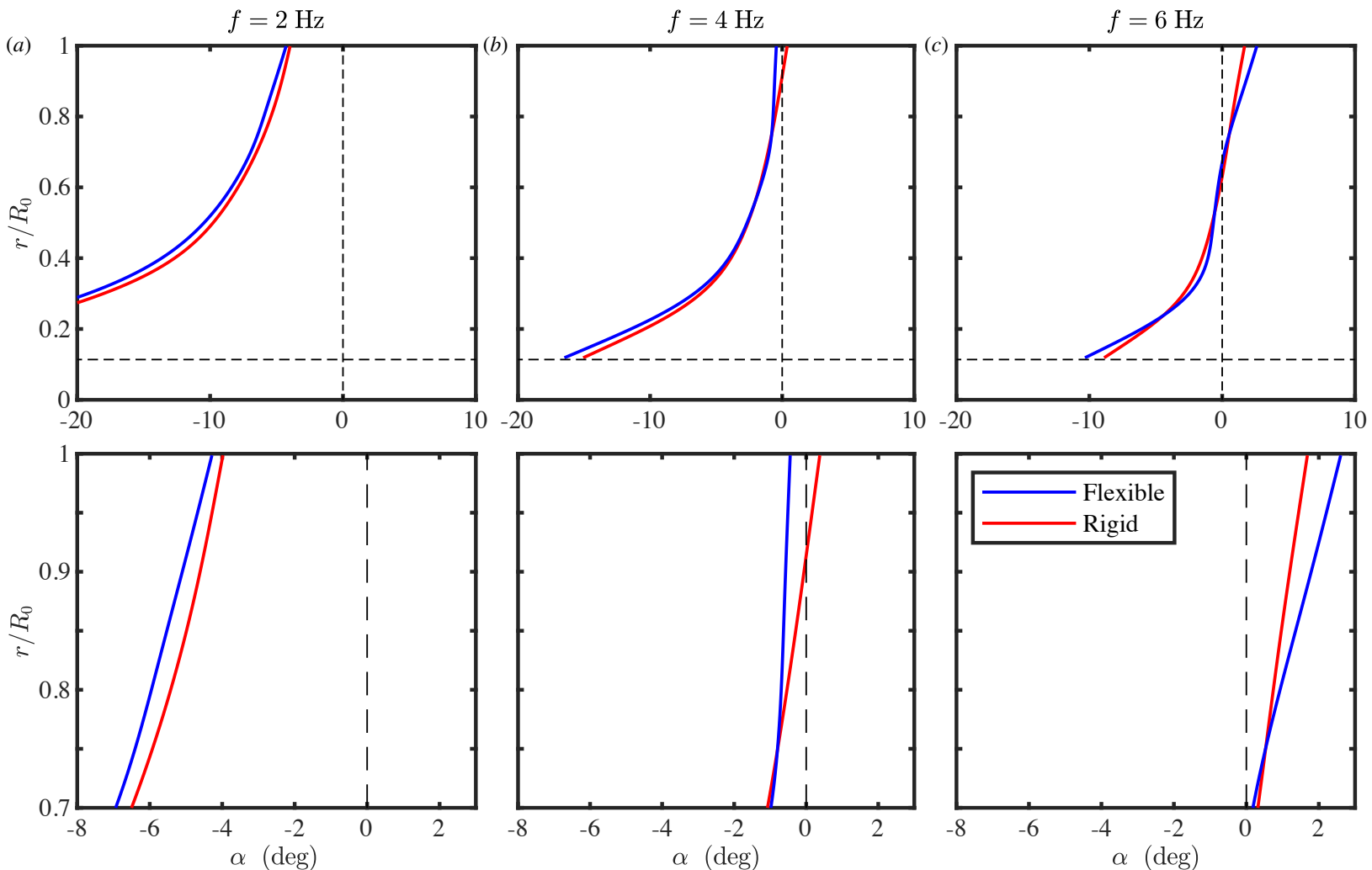

Fig. 19. Comparison of the effective angle of attack profiles for the flexible and rigid blades with pitch $\beta_{0}=5^{\circ}$. (a) $f=2 \mathrm{~Hz}$. (b) $f=4 \mathrm{~Hz}$. (c) $f=6 \mathrm{~Hz}$. The lower plots show close-ups of the tip region.
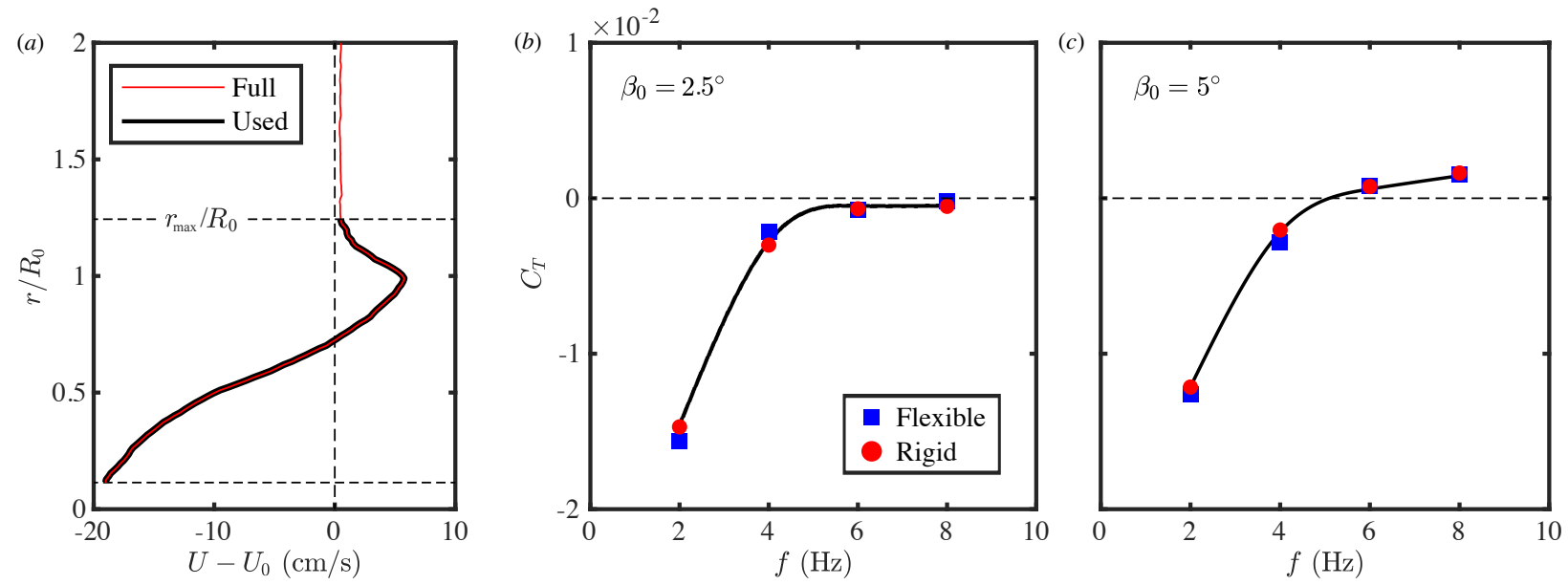

Fig. 20. (a) Radial profile of the time-averaged induced axial velocity, measured at a distance $2 R_{0}$ downstream of the rigid rotor blade with $\beta_{0}=5^{\circ}$ at $f=6 \mathrm{~Hz}$. (b, c) Comparisons of the estimated thrust coefficients for flexible and rigid blades with pitch $\beta_{0}=2.5^{\circ}$ and $\beta_{0}=5^{\circ}$, respectively. 
which decreases in magnitude for higher values. Whereas the thrust of the $\beta_{0}=2.5^{\circ}$ rotor remains negative up to $f=8 \mathrm{~Hz}$, the $\beta_{0}=5^{\circ}$ rotor reverses the thrust into the downstream direction starting at $f \approx 5 \mathrm{~Hz}$. This is consistent with the bending behaviour of the flexible blade described in section 3.1 (Figs. 5, 6 and 8).

The thrust coefficients of the rigid and flexible rotors are nearly the same, the small differences lie within the uncertainty of the measurements. The moderate bending and small twist occurring for the flexible blade do not seem to affect the performance of the rotor in a significant way. Noticeable differences arise when the induced twist is larger, for blades having a much lower torsional stiffness (see, e.g., the study by Mohd-Zawawi, 2014).

\section{Conclusions}

In this study, we have investigated experimentally the fluid-structure interactions of a generic one-bladed rotor, equipped with a moderately flexible blade, in water. The high density of the fluid leads to much larger fluid forces than for similar small-scale rotors operating in air, which can in turn produce significant deformations of the blade. The blade has a rectangular untwisted planform of constant thickness and is tested over a range of pitch angles and rotation rates in a constant free-stream velocity.

For low rotation speed, all blades initially deform in the downstream direction, due to the negative thrust of the rotor. At higher frequency, two different behaviours are found, depending on the blade pitch. At small values, the deformation reaches a maximum, before it decreases again and the blade returns to an almost undeformed state, as the rotation rate further increases. At higher pitch, the blade continues to bend in the downstream direction for negative values, until it reaches extreme deformation amplitudes where the tip almost touches the rotor shaft. For high positive pitch, the initial downstream bending again reaches a maximum with increasing frequency, and then reverses into the upstream direction, since the thrust is inverted for these blades at high rotation. The upstream bending also keeps increasing to extreme values. All high-pitch blades eventually break, when the deformation exceeds the elastic limit of the material.

These observations were characterised quantitatively by measuring the blade deformation from phase-locked image sequences using a suitably defined deflection angle, as well as the blade twist distribution from three-dimensional Digital Image Correlation. Complementary information about the velocity field and vorticity distribution around the rotor was obtained from Particle Image Velocimetry, which allowed the determination of the effective angle of attack of the deformed blade. The analysis of this data showed that the reversal from downstream to upstream bending for blades with high positive pitch is accompanied by a change of sign of the effective angle of attack in the tip region, which leads to an inversion of the induced axial velocity there, and of the tip vortex circulation.

An interesting behaviour was observed in an intermediate frequency range for rotors with negative pitch, for which the blade exhibits bending fluctuations of high amplitude and very low frequency, compared to the rotation rate. Conditional averaging of the wake velocity field, and measurements of the fluctuation frequencies and corresponding Strouhal numbers, show similarities between this phenomenon and the Vortex Ring State encountered in the flow around helicopter rotors in steep descent. The strong negative thrust generates a recirculation zone behind the rotor, taking the form of a large-scale vortex ring, which is shed at regular intervals into the wake, accompanied by variations in the blade position.

Measurements were also carried out for a number of cases using a rigid rotor having the same geometry, in order to assess the effect of the flexibility on the flow field and the rotor performance. It was found that the change of sign of the tip vortex circulation for high positive pitch occurs and a different frequency than for the flexible rotor, which can be explained by the absence of blade twist. For moderate bending and small twist, the estimated thrust coefficients are practically the same for both rigid and flexible rotors in our set-up.

These experiments represent a first exploration of the fluid-structure interaction of a moderately flexible blade rotating in a fluid of high density, which revealed an interesting characteristic deformation behaviour. Further investigations will focus on different regimes of rotor operation, such as hover, by varying the free-stream velocity, which was held constant in the present study. More comparisons of the performance of rigid and flexible rotors, including the regimes of large and unsteady bending, will also be of interest. The modelling of these interactions, which would enable a faster survey of parameter ranges and optimisation studies, presents an additional challenge. The results presented in this paper may help with the validation of such modelling efforts. This study is of fundamental nature, with results covering a wide rang of Reynolds numbers and tip speed ratios, allowing the inclusion of upstream and 
downstream flow induction, which represent different regimes of rotor operation as for helicopters, drones or wind turbines. Direct application of the results from the current blade design to these configurations is hardly possible. Nevertheless, they can be still used as an indicator for the overall behaviour and performance of a moderately flexible blade operating in water or/and air.

\section{Acknowledgements}

The authors are grateful to Eric Bertrand for his assistance with the development of the experimental set-up, and to Alex Montfort for his help with the measurements on rigid blades.

This work was supported by the Excellence Initiative of Aix-Marseille University - A*MIDEX, a French Investissements d'Avenir programme, in the framework of the Labex MEC.

\section{References}

Boden, F., Stasicki, B., 2014. Non-intrusive in-flight propeller blade deformation measurements by means of a rotating camera, in: 34th European Telemetry and Test Conference, pp. 58-63. doi:10.5162/etc2014/4.1.

Calvert, J.R., 1967. Experiments on the flow past an inclined disk. J. Fluid Mech. 29, 691-703. doi:10.1017/S0022112067001120.

Cognet, V., Courrech du Pont, S., Dobrev, I., Massouh, F., Thiria, B., 2017. Bioinspired turbine blades offer new perspectives for wind energy. Proc. R. Soc. A 473, 20160726. doi:10.1098/rspa.2016.0726.

Durán Venegas, E., Le Dizès, S., Eloy, C., 2019. A strongly-coupled model for flexible rotors. J. Fluids Struct. 89, 219-231. doi:10.1016/j . jfluidstructs.2019.03.022.

Grandemange, M., Gohlke, M., Cadot, O., 2013. Bi-stability in the turbulent wake past parallelepiped bodies with various aspect ratios and wall effects. Phys. Fluids 25, 095103. doi:10.1063/1.4820372.

Graves, S.S., Burner, A.W., 2001. Development of an intelligent videogrammetric wind tunnel measurement system. Proc. SPIE 4448, 120-131. doi: $10.1117 / 12.449368$

Hansen, M.O.L., 2015. Aerodynamics of wind turbines. 3rd ed., Routledge.

Herry, B.B., Keirsbulck, L., Labraga, L., Paquet, J.B., 2011. Flow bistability downstream of three-dimensional double backward facing steps at zero-degree sideslip. ASME J. Fluids Eng. 133, 054501. doi:10.1115/1.4004037.

Lawson, N.J., Garry, K.P., Faucompret, N., 2007. An investigation of the flow characteristics in the bootdeck region of a scale model notchback saloon vehicle. Proc. Inst. Mech. Eng. D 221, 739-754. doi:10.1243/09544070JAUT0155.

Leishman, J.G., 2006. Principles of Helicopter Aerodynamics. Cambridge University Press.

Lv, P., Prothin, S., Mohd-Zawawi, F., Bénard, E., Morlier, J., Moschetta, J.M., 2015. Performance improvement of small-scale rotors by passive blade twist control. J. Fluids Struct. 55, 25-41. doi:10.1016/j.jfluidstructs.2015.01.008.

MacPhee, D.W., Beyene, A., 2015. Experimental and fluid structure interaction analysis of a morphing wind turbine rotor. Energy $90,1055-1065$. doi:10.1016/j.energy.2015.08.016.

Medici, D., Alfredsson, P.H., 2006. Measurements on a wind turbine wake: 3D effects and bluff body vortex shedding. Wind Energy $9,219-236$. doi:10.1002/we.156.

Meile, W., Ladinek, T., Brenn, G., Reppenhagen, A., Fuchs, A., 2016. Non-symmetric bi-stable flow around the Ahmed body. Int. J. Heat Fluid Flow 57, 34-47. doi:10.1016/j.ijheatfluidflow.2015.11.002.

Meunier, P., Leweke, T., 2003. Analysis and treatment of errors due to high velocity gradients in particle image velocimetry. Exp. Fluids 35, 408-421. doi:10.1007/s00348-003-0673-2.

Mohd-Zawawi, F., 2014. Aeroelastic analysis of flexible composite proprotor blades for convertible micro air vehicles. Ph.D. thesis. Université de Toulouse. France.

Quaranta, U., 2017. Instabilities in a swirling rotor wake. Ph.D. thesis. Aix-Marseille Université. Marseille, France.

Sicard, J., Sirohi, J., 2012. Experimental study of an extremely flexible rotor for microhelicopters. J. Aircraft 49, 1306-1314. doi:10.2514/1. C031643.

Sicard, J., Sirohi, J., 2013. Measurement of the deformation of an extremely flexible rotor blade using digital image correlation. Meas. Sci. Technol. 24, 65203. doi:10.1088/0957-0233/24/6/065203.

Siddall, R., Kovač, M., 2014. Launching the AquaMAV: bioinspired design for aerial-aquatic robotic platforms. Bioinspir. Biomim. 9, 1-15. doi:10.1088/1748-3182/9/3/031001.

Solav, D., Moerman, K.M., Jaeger, A.M., Genovese, K., Herr, H.M., 2018. MultiDIC: an open-source toolbox for multi-view 3D digital image correlation. IEEE Access 6, 30520-30535. doi:10.1109/ACCESS. 2018.2843725.

Stack, J., Caradonna, F.X., Savaş, O., 2005. Flow visualizations and extended thrust time histories of rotor vortex wakes in descent. J. Am. Helicopter Soc. 50, 279. doi:10.4050/1.3092864.

Sutton, M.A., Orteu, J.J., Schreier, H.W., 2009. Image Correlation for Shape, Motion and Deformation Measurements: Basic Concepts, Theory and Applications. Springer.

Tan, Y.H., Siddall, R., Kovac, M., 2017. Efficient aerial-aquatic locomotion with a single propulsion system. IEEE Robot. Autom. Lett. 2, 13041311. doi:10.1109/LRA.2017.2665689.

Tang, D.M., Dowell, E.H., 1993. Experimental and theoretical study for nonlinear aeroelastic behavior of a flexible rotor blade. AIAA J. 31, 1133-1142. doi:10.2514/3.11738. 
Uehara, D., Sirohi, J., 2019. Full-field optical deformation measurement and operational modal analysis of a flexible rotor blade. Mech. Syst. Signal Process. 133, 106265. doi:10.1016/j.ymssp.2019.106265.

Wald, Q.R., 2006. The aerodynamics of propellers. Progr. Aerosp. Sci. 42, 85-128. doi:10.1016/j.paerosci.2006.04.001.

Winston, M.M., 1968a. A hovering investigation of an extremely flexible lifting rotor. Technical Report TN D-4820. National Aeronautics and Space Administration.

Winston, M.M., 1968b. An investigation of extremely flexible lifting rotors. Technical Report TN D-4465. National Aeronautics and Space Administration.

Yang, X., Wang, T., Liang, J., Yao, G., Liu, M., 2015. Survey on the novel hybrid aquatic-aerial amphibious aircraft: Aquatic unmanned aerial vehicle (AquaUAV). Progr. Aerosp. Sci. 74, 131-151. doi:10.1016/j .paerosci.2014.12.005. 